\title{
Search for Supersymmetry with a Compressed Mass Spectrum in Events with a Soft $\tau$ Lepton, a Highly Energetic Jet, and Large Missing Transverse Momentum in Proton-Proton Collisions at $\sqrt{s}=13 \mathrm{TeV}$
}

\author{
A. M. Sirunyan et al. \\ (CMS Collaboration)
}

(Received 2 October 2019; published 29 January 2020)

\begin{abstract}
The first search for supersymmetry in events with an experimental signature of one soft, hadronically decaying $\tau$ lepton, one energetic jet from initial-state radiation, and large transverse momentum imbalance is presented. These event signatures are consistent with direct or indirect production of scalar $\tau$ leptons $(\tilde{\tau})$ in supersymmetric models that exhibit coannihilation between the $\tilde{\tau}$ and the lightest neutralino $\left(\tilde{\chi}_{1}^{0}\right)$, and that could generate the observed relic density of dark matter. The data correspond to an integrated luminosity of $77.2 \mathrm{fb}^{-1}$ of proton-proton collisions at $\sqrt{s}=13 \mathrm{TeV}$ collected with the CMS detector at the LHC in 2016 and 2017. The results are interpreted in a supersymmetric scenario with a small mass difference $(\Delta m)$ between the chargino $\left(\tilde{\chi}_{1}^{ \pm}\right)$or next-to-lightest neutralino $\left(\tilde{\chi}_{2}^{0}\right)$, and the $\tilde{\chi}_{1}^{0}$. The mass of the $\tilde{\tau}$ is assumed to be the average of the $\tilde{\chi}_{1}^{ \pm}$and $\tilde{\chi}_{1}^{0}$ masses. The data are consistent with standard model background predictions. Upper limits at $95 \%$ confidence level are set on the sum of the $\tilde{\chi}_{1}^{ \pm}, \tilde{\chi}_{2}^{0}$, and $\tilde{\tau}$ production cross sections for $\Delta m\left(\tilde{\chi}_{1}^{ \pm}, \tilde{\chi}_{1}^{0}\right)=50 \mathrm{GeV}$, resulting in a lower limit of $290 \mathrm{GeV}$ on the mass of the $\tilde{\chi}_{1}^{ \pm}$, which is the most stringent to date and surpasses the bounds from the LEP experiments.
\end{abstract}

DOI: 10.1103/PhysRevLett.124.041803

Supersymmetry (SUSY) [1-7] is a theoretical extension of the standard model (SM) that could describe the particle nature of dark matter (DM) and solve the gauge hierarchy problem. In SUSY models assuming $\mathcal{R}$ parity [8] conservation, if the lightest neutralino $\left(\tilde{\chi}_{1}^{0}\right)$ is the lightest SUSY particle, it is neutral, stable, and could have undergone annihilation-production interactions with SM particles in the early universe to give the DM relic density observed today $[9,10]$. In models with a bino (Z-like) $\tilde{\chi}_{1}^{0}$, these interactions alone are insufficient to produce the correct DM relic abundance. As such, a model of coannihilation (CA) can be introduced, where CA refers to the interaction of $\tilde{\chi}_{1}^{0}$ with another SUSY particle resulting in the production of SM particles [11].

This Letter describes a search for the production of stau particles $(\tilde{\tau})$, SUSY partners of the $\tau$ lepton, considering a mass difference $(\Delta m)$ between the $\tilde{\chi}_{1}^{0}$ and $\tilde{\tau}$ of $\leq 50 \mathrm{GeV}$. These scenarios are motivated by models including $\tilde{\tau}-\tilde{\chi}_{1}^{0}$ CA [12-19], where the calculated relic DM density is consistent with that measured by the WMAP and Planck

\footnotetext{
*Full author list given at the end of the article.

Published by the American Physical Society under the terms of the Creative Commons Attribution 4.0 International license. Further distribution of this work must maintain attribution to the author(s) and the published article's title, journal citation, and DOI. Funded by SCOAP ${ }^{3}$.
}

Collaborations $[9,10]$. The CA cross section is exponentially enhanced by small $\Delta m\left(\tilde{\tau}, \tilde{\chi}_{1}^{0}\right)$.

In proton-proton $(p p)$ collisions at the LHC, $\tilde{\tau}$ particles can be produced directly in pairs or in decays of heavier SUSY particles. The $\tilde{\tau}$ can decay to a $\tau$ lepton and $\tilde{\chi}_{1}^{0}$. The analysis described in this Letter requires an extra jet $(j)$ from initial-state radiation (ISR). The recoil effect from the ISR jet facilitates the detection of momentum imbalance and identification of the low-energy (soft) $\tau$ lepton decay products [18-26]. Thus, this analysis focuses on $p p \rightarrow \tilde{\tau} \tilde{\tau} \mathrm{j}$ production and indirect $\tilde{\tau}$ production via decays of the lightest chargino $\left(\tilde{\chi}_{1}^{ \pm}\right)$or the next-to-lightest neutralino $\left(\tilde{\chi}_{2}^{0}\right)$ in processes like $p p \rightarrow \tilde{\chi}_{1}^{ \pm} \chi_{1}^{\mp} \mathrm{j} \rightarrow \tilde{\tau} \tilde{\tau} \nu \tau \nu \tau \mathrm{j} \rightarrow$ $\tau \tilde{\chi}_{1}^{0} \tau \tilde{\chi}_{1}^{0} \nu \tau \nu \tau \mathrm{j}$ and $p p \rightarrow \tilde{\chi}_{1}^{ \pm} \tilde{\chi}_{2}^{0} \mathrm{j} \rightarrow \tilde{\tau} \nu \tau \tilde{\tau} \tau \mathrm{j} \rightarrow \tau \tilde{\chi}_{1}^{0} \nu \tau \tau \tilde{\chi}_{1}^{0} \tau \mathrm{j}$, which can be the dominant production mechanisms for $\tilde{\tau}$ via decays of heavier SUSY particles. While these processes yield final states with multiple $\tau$ leptons, the average transverse momentum $\left(p_{\mathrm{T}}\right)$ of the $\tau$ leptons is $\Delta m / 2$ and below the reconstruction threshold in the $\Delta m\left(\tilde{\tau}, \tilde{\chi}_{1}^{0}\right) \leq 50 \mathrm{GeV}$ scenarios. The visible decay products of the $\tau$ leptons have lower $p_{\mathrm{T}}$ than the decaying particles, so it is difficult to identify more than one $\tau$ lepton in a signal event. Furthermore, leptonic decays of $\tau$ leptons have a smaller branching fraction $(\mathcal{B})$ than hadronic decays $\left(\tau_{\mathrm{h}}\right)$, and, on average, smaller visible $p_{\mathrm{T}}$. Electrons and muons from such decays are also indistinguishable from prompt production of electrons and muons. Hence, we search for events with exactly one soft 
$\tau_{\mathrm{h}}$ candidate and missing transverse momentum recoiling against a high- $p_{\mathrm{T}}$ ISR jet.

The strategy above allows this analysis to probe the $\tilde{\tau}-\tilde{\chi}_{1}^{0}$ CA region with $\Delta m\left(\tilde{\tau}, \tilde{\chi}_{1}^{0}\right) \leq 50 \mathrm{GeV}$. This is the first collider search for compressed SUSY spectra using this strategy. Earlier searches from the CMS and ATLAS Collaborations [27-33] that relate to the scenarios in this Letter produced weaker results than those from the LEP experiments [34-37]. Data collected in 2016 and 2017 with the CMS experiment [38] in $p p$ collisions at $\sqrt{s}=13 \mathrm{TeV}$ is used. The data sample corresponds to an integrated luminosity of $77.2 \mathrm{fb}^{-1}$.

The central feature of the CMS apparatus [38] is a superconducting solenoid of $6 \mathrm{~m}$ internal diameter, providing a magnetic field of $3.8 \mathrm{~T}$. Within the solenoid volume are a silicon pixel and strip tracker, a lead tungstate crystal electromagnetic calorimeter (ECAL), and a brass and scintillator hadron calorimeter, each composed of a barrel and two endcap sections. Forward calorimeters extend the pseudorapidity $(\eta)$ coverage of the barrel and endcap detectors up to $|\eta|<5.2$. Muons are measured in gasionization detectors embedded in the steel flux-return yoke outside the solenoid. A detailed description of the CMS detector can be found in Ref. [38].

Events are reconstructed from particle candidates (electrons, muons, photons, and hadrons) identified using the particle-flow (PF) algorithm [39]. The algorithm combines information from all detectors to classify final-state particles produced in the collision. Jets are clustered using the anti- $k_{\mathrm{T}}$ clustering algorithm $[40,41]$ with a distance parameter of 0.4. Identification criteria are applied to jet candidates to remove anomalous effects from the calorimeters [42]. For jets with $p_{\mathrm{T}}>30 \mathrm{GeV}$ and $|\eta|<2.4$, the identification efficiency is $>99 \%$ [43].

The jet energy scale and resolution are corrected depending on the $p_{\mathrm{T}}$ and $\eta$ of the jet [44]. Jets originating from the hadronization of $b$ quarks are identified using the combined secondary vertex algorithm [45]. This analysis uses the loose working point of the algorithm, which has an identification efficiency of $80 \%$ for $b$ jets and a lightflavor quark or gluon misidentification rate of $10 \%$.

Electrons and muons are used in control samples and as vetoes in the signal sample selection. Electrons are reconstructed and identified combining information from the ECAL and the tracking system [46]. Muons are reconstructed using the tracker and muon chambers, and requiring consistency with low-energy measurements in the calorimeters [47]. For this analysis, the electron (muon) identification efficiency is 85 (96)\%, for leptons with $p_{\mathrm{T}}>10 \mathrm{GeV}$ and $|\eta|<2.1$.

Hadronic decays of $\tau$ leptons are reconstructed and identified using the hadrons-plus-strips algorithm [48], designed to optimize $\tau_{\mathrm{h}}$ reconstruction by considering specific $\tau_{\mathrm{h}}$ decay modes. To suppress backgrounds from light-flavor quark or gluon jets, $\tau_{\mathrm{h}}$ candidates are required to pass a threshold value of a multivariate discriminator that takes variables related to isolation and $\tau$ lepton lifetime as input. The tight isolation working point is used, which results in a $\tau_{\mathrm{h}}$ identification efficiency of $55 \%$ for this analysis, and a $0.2-5 \%$ probability for a jet to be misidentified as a $\tau_{\mathrm{h}}$, depending on the $p_{\mathrm{T}}$ and $\eta$ values of the $\tau_{\mathrm{h}}$ candidate [48]. The $\tau_{\mathrm{h}}$ candidates are subject to additional requirements, based on consistency among measurements in the tracker, calorimeters, and muon detectors, to distinguish them from electrons and muons.

The missing transverse momentum $\vec{p}_{\mathrm{T}}^{\text {miss }}$ is the negative vector $p_{\mathrm{T}}$ sum of all $\mathrm{PF}$ candidates. Its magnitude is $p_{\mathrm{T}}^{\text {miss }}$. Production of undetected particles such as SM neutrinos and the $\tilde{\chi}_{1}^{0}$ is inferred from the measured $p_{\mathrm{T}}^{\text {miss }}[49,50]$. The jet corrections described are propagated as corrections to $p_{\mathrm{T}}^{\text {miss }}$, which improves agreement in $p_{\mathrm{T}}^{\text {miss }}$ between simulation and data.

The dominant SM background processes contributing to the search are $W / Z$ boson production in association with jets $(W+$ jets and $Z+$ jets), top quark pairs $(t \bar{t})$, and quantum chromodynamics (QCD) multijet processes. The contributions of $W+$ jets and $Z+$ jets events contain genuine $\tau_{\mathrm{h}}$ candidates, energetic jets, and $p_{\mathrm{T}}^{\text {miss }}$ from neutrinos. Background from $t \bar{t}$ events is characterized by two $b$ quark jets in addition to a genuine $\tau_{\mathrm{h}}$. QCD multijet events are characterized by jets misidentified as $\tau_{\mathrm{h}}$, and the estimated yield of this background is derived from data.

Simulated samples for $Z+$ jets, $W+$ jets, $t \bar{t}+$ jets, and single top quark events are produced with the MADGRAPH 5_aMC@NLO 2.6.0 program [51] at leading order (LO) precision. The LO PYTHIA generator is used to model diboson (VV) processes. Two sets of signal event samples are generated using MADGRAPH 5_aMC@NLO 2.3.3 at LO precision. The first set considers the sum of $\tilde{\chi}_{1}^{ \pm} \chi_{1}^{\mp}, \tilde{\chi}_{1}^{ \pm} \tilde{\chi}_{2}^{0}$, and $\tilde{\tau} \tilde{\tau}$ production with up to two jets. The $\tilde{\tau} \tilde{\tau}$ process represents $<2 \%$ of the total cross section. Models with a bino $\tilde{\chi}_{1}^{0}$ and wino ( $W$-like) $\tilde{\chi}_{2}^{0}$ and $\tilde{\chi}_{1}^{ \pm}$are considered. We assume a simplified model scenario [52] with a left-handed $\tilde{\tau}, \mathcal{B}\left(\tilde{\chi}_{2}^{0} \rightarrow \tau \tilde{\tau} \rightarrow \tau \tau \tilde{\chi}_{1}^{0}\right)=\mathcal{B}\left(\tilde{\chi}_{1}^{ \pm} \rightarrow \nu_{\tau} \tilde{\tau} \rightarrow \nu_{\tau} \tau \tilde{\chi}_{1}^{0}\right)=100 \%$, and $m\left(\tilde{\chi}_{1}^{ \pm}\right)=m\left(\tilde{\chi}_{2}^{0}\right)$. This set of samples is motivated by the importance of the chargino-neutralino sector in connecting SUSY models and DM. We refer to this model as SUSY signal model 1 (SSM1). The second set considers direct production of left-handed $\tilde{\tau}$ pairs with up to two jets. Although the search for direct $\tilde{\tau}$ production with $\Delta m\left(\tilde{\tau}, \tilde{\chi}_{1}^{0}\right) \leq 50 \mathrm{GeV}$ is challenging because of the small production cross section and low signal acceptance, this set of samples is included to highlight the improved sensitivity in this analysis, compared to previous non-ISR searches [31,34-37,53-57]. This second set of samples allows for reinterpretation in other scenarios with $\tilde{\tau}$-like particles. We refer to this model as SUSY signal model 2 (SSM2). It is noted that the masses of $\tilde{\chi}_{1}^{ \pm} / \tilde{\chi}_{2}^{0}$ are sufficiently large $(10 \mathrm{TeV})$ to be considered decoupled in SSM2. 
The MADGRAPH 5_aMC@NLO generator is interfaced with PYTHIA 8.212 [58] using the CUETP8M1 and CP5 tunes $[59,60]$ for parton shower and fragmentation in the 2016 and 2017 simulated samples, respectively. The NNPDF3.0 LO and NLO [61] parton distribution functions (PDFs) are used in the event generation. The CMS detector response is simulated using the GEANT4 [62] package for background samples, and the CMS fast simulation package [63] for signal samples. To model the effect of additional $p p$ interactions within the same bunch crossing or nearby bunch crossings, minimum bias events generated with PYTHIA are added to the simulated samples with a frequency distribution per bunch crossing weighted to match that observed in data. MC background yields are normalized to the integrated luminosity using next-to-next-toleading order (NNLO) or next-to-leading order (NLO) cross sections, while signal production cross sections are calculated at NLO with next-to-leading logarithmic (NLL) soft-gluon resummation calculations [64-67].

Events are recorded using a $p_{\mathrm{T}}^{\text {miss }}$ trigger [68]. The trigger efficiency is measured using data events with one muon, resulting in a sample enriched in $W+$ jets events $(95 \%$ purity in simulation). Selected events are required to have $p_{\mathrm{T}}^{\text {miss }}>230 \mathrm{GeV}$, where the trigger is fully efficient, and exactly one identified $\tau_{\mathrm{h}}$ candidate with $|\eta|<2.1$ and $20<p_{\mathrm{T}}\left(\tau_{\mathrm{h}}\right)<40 \mathrm{GeV}$. The requirement of exactly one $\tau_{\mathrm{h}}$ candidate and the upper limit on $p_{\mathrm{T}}$ reduce the $W+$ jets, $Z+$ jets, and $t \bar{t}+$ jets backgrounds. The highest- $p_{\mathrm{T}}$ jet is referred to as the ISR jet ( $\mathrm{j}_{\text {ISR }}$ ) and is required to satisfy $p_{\mathrm{T}}>100 \mathrm{GeV}$ and $|\eta|<2.4$. The absolute difference in the azimuthal angle $(\phi)$ between the ISR jet and $\vec{p}_{\mathrm{T}}^{\text {miss }}$ is required to be greater than 0.7 radians $\left(\left|\Delta \phi\left(\mathrm{j}_{\mathrm{ISR}}, \vec{p}_{\mathrm{T}}^{\text {miss }}\right)\right|>0.7\right.$ radians) to reduce QCD multijet events containing large $p_{\mathrm{T}}^{\text {miss }}$ from jet mismeasurements. To reduce background processes with top quarks, events with $b$-tagged jets are rejected. Events with well-identified and isolated electrons or muons with $p_{\mathrm{T}}>10 \mathrm{GeV}$ and $|\eta|<2.1$ are rejected.

The transverse mass of the selected $\tau_{\mathrm{h}}$ candidate and the $\vec{p}_{\mathrm{T}}^{\text {miss }}$, defined as

$m_{\mathrm{T}}\left(\vec{p}_{\mathrm{T}}^{\text {miss }}, \tau_{\mathrm{h}}\right)=\sqrt{2 p_{\mathrm{T}}^{\text {miss }} p_{\mathrm{T}}\left(\tau_{\mathrm{h}}\right)\left[1-\cos \Delta \phi\left(\vec{p}_{\mathrm{T}}^{\text {miss }}, \tau_{\mathrm{h}}\right)\right]}$,

is the main observable to search for the presence of signal events. The $m_{\mathrm{T}}$ in signal events probes the SUSY mass scale, and is expected to be larger on average than for the backgrounds. The strategy is to search for a broad enhancement in the high- $m_{\mathrm{T}}$ part of the spectrum.

The yield and $m_{\mathrm{T}}$ shape of the QCD multijet background are estimated from data using control regions (CRs) enriched in QCD multijet events and with negligible signal contamination. MC simulations are used to extrapolate the $W / Z+$ jets and $t \bar{t}+$ jets background yields from a CR to the signal region (SR) and to model $m_{\mathrm{T}}$ shapes. The agreement between data and simulation in these CRs is used to validate the modeling of the $\tau_{\mathrm{h}}$ selections and to measure data-to-simulation scale factors to correct the modeling of the ISR jet and the $p_{\mathrm{T}}^{\text {miss }}$. To calculate the correction factor, contributions from nontargeted backgrounds are subtracted from data. The uncertainty in these background processes is propagated to the final systematic uncertainty in the background predictions. Small contributions from single top quark and diboson production are estimated using simulation.

The correct modeling in the simulation of background events, in particular the $W / Z+$ jets processes, can be affected by requiring an ISR jet. This modeling is studied using a $Z(\rightarrow \mu \mu)+$ jets $\mathrm{CR}$ in data. This CR provides a measurement of the $p_{\mathrm{T}}$ spectrum resulting from a high- $p_{\mathrm{T}}$ ISR jet, decoupling the effects of ISR modeling from the measurement of $p_{\mathrm{T}}^{\text {miss }}$. The $p_{\mathrm{T}}$ of the $Z$ boson is measured by vectorially summing the transverse momenta of the two muons from the $Z$ decay. The ratio between data and simulation in the $p_{\mathrm{T}}(\mu \mu)$ distribution is used to obtain $p_{\mathrm{T}}$-dependent correction factors, ranging from 0.79 to 1.12 . The factors are validated using a $W\left(\rightarrow \mu \nu_{\mu}\right)+$ jets enriched sample. After applying these correction factors, we find agreement between the observed and predicted yields and shapes of distributions. These ISR correction factors are applied to all Drell-Yan processes, including the $W / Z+$ jets backgrounds and signal processes.

A $Z\left(\rightarrow \tau_{\mathrm{h}} \tau_{\mathrm{h}}\right)+$ jets $\mathrm{CR}$ is defined to study the modeling of $\tau_{\mathrm{h}}$ reconstruction and identification. The CR is obtained by requiring two $\tau_{\mathrm{h}}$ candidates with $p_{\mathrm{T}}>60 \mathrm{GeV}$ and $|\eta|<2.1$, selected by a dedicated $\tau_{\mathrm{h}} \tau_{\mathrm{h}}$ trigger [31,69-71]. The two $\tau_{\mathrm{h}}$ candidates of a pair must have opposite electric charge and a reconstructed mass between 50 and $100 \mathrm{GeV}$, and all other requirements are the same as for SR events. The contribution of QCD multijet events in the $Z\left(\rightarrow \tau_{\mathrm{h}} \tau_{\mathrm{h}}\right)+$ jets $\mathrm{CR}$ is estimated from data using CRs obtained with $\tau_{\mathrm{h}}$ pairs with the same electric charge. The transfer factor between same- and opposite-sign events is calculated using events with loosened $\tau_{\mathrm{h}}$ isolation requirements and $m\left(\tau_{\mathrm{h}} \tau_{\mathrm{h}}\right)>100 \mathrm{GeV}$. Correction factors of $0.92 \pm 0.05$ and $0.95 \pm 0.04$ for $Z\left(\rightarrow \tau_{\mathrm{h}} \tau_{\mathrm{h}}\right)+$ jets are measured in this CR for the 2016 and 2017 data sets, respectively. The uncertainties are purely statistical. These correction factors are used to scale the $Z(\rightarrow \tau \tau)+$ jets prediction in the SR.

The contribution from $t \bar{t}$ events in the SR is less than 15\% of the total expected background. Correction factors of $0.94 \pm 0.05$ and $0.95 \pm 0.04$ are measured for the 2016 and 2017 data sets, respectively, in a CR obtained by selecting events with two $b$-tagged jets and one $\tau_{\mathrm{h}}$ candidate with tighter isolation requirements with respect to the SR. These requirements allow for a $t \bar{t} \mathrm{CR}$ sample with high purity. The correction factor is applied to scale the prediction of $t \bar{t}$ events in the SR.

A CR enriched with $\mathrm{QCD}$ multijet events $\left(\mathrm{CR}_{\mathrm{QCD}}\right)$ is obtained by requiring the same criteria for the SR but 
selecting $\tau_{\mathrm{h}}$ candidates that fail the tight and pass the loose $\tau_{\mathrm{h}}$ isolation. The contribution from nonmultijet events is subtracted using simulation, adjusted for the scale factors discussed above. The shape and normalization of the multijet background in the SR are predicted by multiplying the data yields in $\mathrm{CR}_{\mathrm{QCD}}$ with transfer factors ("tight-toloose" ratios) to account for the isolation efficiency. The $p_{\mathrm{T}}\left(\tau_{\mathrm{h}}\right)$-dependent transfer factors are derived in a $W\left(\rightarrow \mu \nu_{\mu}\right)+\tau_{\mathrm{h}}$ CR, where the $\tau_{\mathrm{h}}$ is a misidentified jet. These transfer factors, which range from 0.2 to 0.4 , are validated in a region enriched in QCD multijet events by inverting the $\Delta \phi\left(\mathrm{j}_{\mathrm{ISR}}, \vec{p}_{\mathrm{T}}^{\text {miss }}\right)$ requirement.

A major source of systematic uncertainty is the closure of the background estimation methods, where closure refers to tests (on data and simulation) which demonstrate that the background determination techniques reproduce the expected background distributions in both rate and shape within the statistical uncertainties. The background estimation uncertainty from the closure tests is $2-6 \%$ for nonmultijet backgrounds. For the QCD multijet background, this uncertainty is determined by the deviation of the tight-to-loose ratios obtained in a $Z(\rightarrow \mu \mu)+\tau_{\mathrm{h}} \mathrm{CR}$, where the $\tau_{\mathrm{h}}$ is a misidentified jet, from those in the $W\left(\rightarrow \mu \nu_{\mu}\right)+\tau_{\mathrm{h}}$ region. This uncertainty depends on $p_{\mathrm{T}}\left(\tau_{\mathrm{h}}\right)$ and varies from 4 to $29 \%$. Shape-based systematic uncertainties from the use of ISR correction factors are determined by varying these factors by \pm 1 standard deviation of their uncertainty and examining effects on the $m_{\mathrm{T}}$ distribution. This uncertainty is a few percent at low $m_{\mathrm{T}}$ and $15 \%$ at high $m_{\mathrm{T}}$. Although the corrected background $m_{\mathrm{T}}$ shapes are consistent with the data distributions within statistical uncertainties, data-to-simulation ratios of the $m_{\mathrm{T}}$ distributions are fit with a first-order polynomial, and the deviation of the fit from unity, as a function of $m_{\mathrm{T}}$, is taken as an uncertainty in the shape. This results in up to $20 \%$ uncertainty in a given $m_{\mathrm{T}}$ bin.

The signal and background yields estimated from simulation are affected by similar sources of systematic uncertainty, with small differences between the 2016 and 2017 data sets. The uncertainty from the $\tau_{\mathrm{h}}$ identification and isolation requirements ranges between 6 and $9 \%$, depending on the year and process [48]. Efficiencies for the electron and muon reconstruction, identification, and isolation requirements are considered because of the extra lepton vetoes in the SR and their use in the CRs $[46,47,72]$, with an uncertainty of $\leq 1 \%$. The $p_{\mathrm{T}}^{\text {miss }}$ scale uncertainties due to the jet energy scale (2-5\% depending on $\eta$ and $p_{\mathrm{T}}$ ) result in an uncertainty of $1-3 \%$ depending on $m_{\mathrm{T}}$. The event acceptance for the ISR selection depends on the reconstruction and identification efficiencies and the energy scale of jets. A $p_{\mathrm{T}}^{\text {miss }}$-dependent uncertainty in the measured trigger efficiency results in a $3 \%$ uncertainty. The uncertainty in event acceptance from the PDF set used in simulation is evaluated in accordance with the PDF4LHC recommendations [73] by comparing results using the CTEQ6.6L, MSTW08, and

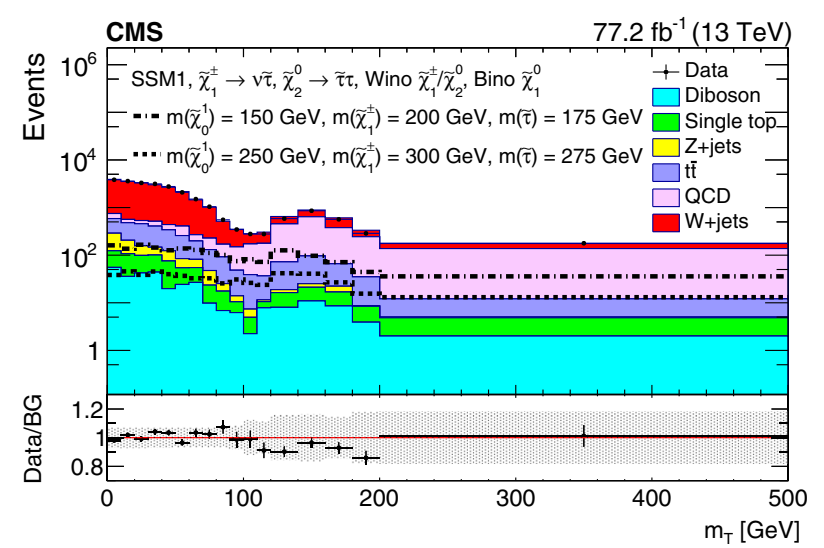

FIG. 1. The $m_{\mathrm{T}}$ distribution for SR events with 2016 plus 2017 data. In the upper panel, the solid colors correspond to the expected background processes, the black dots to the observed data, and the dashed lines to the expected signal from simulation. The lower panel shows the ratio between the observed data and the total expected pre-fit background (BG). The shaded band corresponds to the total pre-fit uncertainty on the BG prediction, while the error bars on the black dots represent the statistical uncertainties on the data yields.

NNPDF10 PDF sets [74-76] with those from the default PDF set. A systematic uncertainty in the signal accounts for differences between the fast and GEANT4 simulations, which depends on $m_{\mathrm{T}}$ and varies from 3 to $11 \%$. The uncertainty in the integrated luminosity corresponds to 2.5 [77] and $2.3 \%$ [78] for the 2016 and 2017 data, respectively.

Figure 1 shows the $m_{\mathrm{T}}\left(p_{\mathrm{T}}^{\text {miss }}, \tau_{\mathrm{h}}\right)$ distribution for events in the SR. The binning used in Fig. 1 is optimized to achieve the best discovery potential for the SSM1 scenarios, resulting in bins of $10 \mathrm{GeV}$ width between $m_{\mathrm{T}}$ of 0 and $120 \mathrm{GeV}$, bins of $20 \mathrm{GeV}$ width between $m_{\mathrm{T}}$ of 120 and $200 \mathrm{GeV}$, and one bin of $300 \mathrm{GeV}$ width for $m_{\mathrm{T}}>200 \mathrm{GeV}$. For a SSM1 benchmark sample with $m\left(\tilde{\chi}_{1}^{ \pm}\right)=200 \mathrm{GeV}, m(\tilde{\tau})=175 \mathrm{GeV}$, and $m\left(\tilde{\chi}_{1}^{0}\right)=150 \mathrm{GeV}$, the signal-to-background ratio ranges from $\approx 1 / 25$ at low $m_{\mathrm{T}}$ to $\approx 1 / 3$ at high $m_{\mathrm{T}}$.

No significant excess above the background prediction is observed. The 95\% confidence level (C.L.) upper limits are set on the SSM1 signal production cross sections as a function of $m\left(\tilde{\chi}_{1}^{ \pm}\right)$for fixed $\Delta m\left(\tilde{\chi}_{1}^{ \pm}, \tilde{\chi}_{1}^{0}\right)=50 \mathrm{GeV}$ and $m(\tilde{\tau})=0.5 m\left(\tilde{\chi}_{1}^{ \pm}\right)+0.5 m\left(\tilde{\chi}_{1}^{0}\right)$ (Fig. 2 left). This benchmark is motivated by: (i) LHC searches to date have no sensitivity in these SSM1 compressed spectrum scenarios; and (ii) SSM1 scenarios with $\Delta m\left(\tilde{\tau}, \tilde{\chi}_{1}^{0}\right)=25 \mathrm{GeV}$ provide the right $\mathrm{CA}$ cross section to give a DM relic density consistent with experiment [12-19]. Figure 2 right shows the observed 95\% C.L. upper limits on the SSM2 signal production cross sections as a function of $m(\tilde{\tau})$ and $\Delta m\left(\tilde{\tau}, \tilde{\chi}_{1}^{0}\right)$. The limits are estimated following the modified frequentist construction $\mathrm{CL}_{\mathrm{s}}$ method [79-81]. Maximum likelihood fits are performed using the final $m_{\mathrm{T}}$ distributions for 2016 and 2017 data to construct a combined profile likelihood ratio test statistic [79] in bins of $m_{\mathrm{T}}$. 

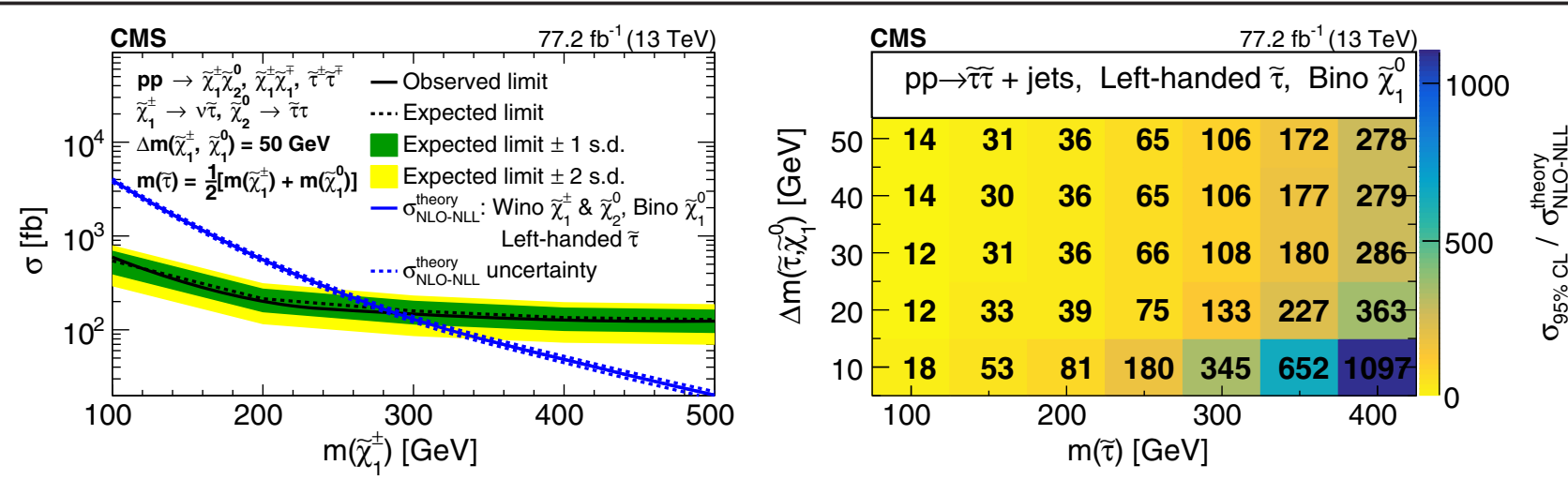

FIG. 2. (left) The $95 \%$ confidence level (C.L.) upper limits on the SSM1 production cross sections $\left(\sigma_{95 \% \text { C.L. }}\right)$ as a function of $m\left(\tilde{\chi}_{1}^{ \pm}\right)$. The solid blue line shows the theoretical cross section, and the dashed blue line its uncertainty. The observed limit is shown with the solid black line, while the expected limit is shown with the dashed black line. The green (yellow) band corresponds to the one (two) standard deviation range about the central value of the expected limit. (right) The ratio of the 95\% C.L. upper limit on the direct $\tilde{\tau}$ pair production signal cross section in SSM2 to the theoretical cross section, as a function of $m(\tilde{\tau})$ and $\Delta m\left(\tilde{\tau}, \tilde{\chi}_{1}^{0}\right)$.

Systematic uncertainties are represented by nuisance parameters, assuming log-normal priors for normalization parameters, and Gaussian priors for shape uncertainties. Statistical uncertainties in the shape templates are accounted for by the technique described in Ref. [82]. Correlations among the signal and backgrounds have been considered. For example, the uncertainty in the integrated luminosity is treated as fully correlated across signal and backgrounds, while uncertainties from event acceptance variation with different sets of PDFs or variations in the ISR correction factors, in a given $m_{\mathrm{T}}$ bin, are treated as uncorrelated. Uncertainties from the closure tests are treated as uncorrelated. We note that the statistical uncertainty dominates the sensitivity.

For SSM1, we exclude $\tilde{\chi}_{2}^{0} / \tilde{\chi}_{1}^{ \pm}$with masses below $290 \mathrm{GeV}$ for $\Delta m\left(\tilde{\chi}_{1}^{ \pm}, \tilde{\chi}_{1}^{0}\right)=50 \mathrm{GeV}$ and $\Delta m\left(\tilde{\chi}_{1}^{ \pm}, \tilde{\tau}\right)=$ $25 \mathrm{GeV}$. Prior experimental constraints on the SUSY parameters with these $\Delta m\left(\tilde{\chi}_{1}^{ \pm}, \tilde{\chi}_{1}^{0}\right)$ and $\Delta m\left(\tilde{\chi}_{1}^{ \pm}, \tilde{\tau}\right)$ values using non-ISR searches [27-32] have not exceeded those of the LEP experiments for indirect $\tilde{\tau}$ production [34-37]. Thus the search presented in this Letter provides the first results from the LHC to surpass the LEP bound of 103.5 $\mathrm{GeV}$ for $m\left(\tilde{\chi}_{1}^{ \pm}\right)$for such compressed scenarios. For SSM2, small $\tilde{\tau} \tilde{\tau}$ production cross sections and low signal acceptances make these scenarios challenging, especially when $\Delta m\left(\tilde{\tau}, \tilde{\chi}_{1}^{0}\right) \leq 50 \mathrm{GeV}$. For a $\tilde{\tau}$ mass of $100 \mathrm{GeV}$ and $\Delta m\left(\tilde{\tau}, \tilde{\chi}_{1}^{0}\right)=30 \mathrm{GeV}$, for example, the observed limit is 12 times the theoretical cross section. It is again noted that the SSM2 results are included in this Letter to highlight the improved sensitivity in this analysis compared to previous non-ISR searches. A direct comparison with the most sensitive non-ISR search, Ref. [57], shows $\approx \times 4$ improvement in the cross section upper limit for the SSM2 scenario with $m(\tilde{\tau})=150 \mathrm{GeV}$ and $\Delta m\left(\tilde{\tau}, \tilde{\chi}_{1}^{0}\right)=50 \mathrm{GeV}$.

In summary, we have presented a search for compressed supersymmetry. It is the first collider search with exactly one soft, hadronically-decaying tau lepton and missing transverse momentum recoiling against an initial-state radiation jet with high transverse momentum. The search utilizes data corresponding to an integrated luminosity of $77.2 \mathrm{fb}^{-1}$ collected with the CMS detector in proton-proton collisions at $\sqrt{s}=13 \mathrm{TeV}$. This search targets scenarios where the mass difference $(\Delta m)$ between the stau $(\tilde{\tau})$ particle and the lightest neutralino $\left(\tilde{\chi}_{1}^{0}\right)$ is $\leq 50 \mathrm{GeV}$. This is motivated by models considering $\tilde{\tau}-\tilde{\chi}_{1}^{0}$ CA to maintain consistency in the relic DM density between particle physics and cosmology. In the context of the minimal supersymmetric standard model, the search considers electroweak production of $\tilde{\tau}$ via decays of the lightest chargino $\left(\tilde{\chi}_{1}^{ \pm}\right)$and the next-to-lightest neutralino $\left(\tilde{\chi}_{2}^{0}\right)$, and direct production of $\tilde{\tau}$. The data do not reveal evidence for new physics. For a mass splitting $\Delta m\left(\tilde{\chi}_{1}^{ \pm}, \tilde{\chi}_{1}^{0}\right)=50 \mathrm{GeV}$ and a branching fraction of $100 \%$ for $\tilde{\chi}_{1}^{ \pm} \rightarrow \tilde{\tau} \nu_{\tau} \rightarrow \tau \tilde{\chi}_{1}^{0} \nu_{\tau}$, $\tilde{\chi}_{1}^{ \pm}$masses up to $290 \mathrm{GeV}$ are excluded at $95 \%$ confidence level. This sensitivity exceeds that of all other $\tilde{\tau}$ searches to date in these scenarios. The search presented in this Letter provides the first results from the LHC to surpass the LEP bounds.

We congratulate our colleagues in the CERN accelerator departments for the excellent performance of the LHC and thank the technical and administrative staffs at CERN and at other CMS institutes for their contributions to the success of the CMS effort. In addition, we gratefully acknowledge the computing centers and personnel of the Worldwide LHC Computing Grid for delivering so effectively the computing infrastructure essential to our analyses. Finally, we acknowledge the enduring support for the construction and operation of the LHC and the CMS detector provided by the following funding agencies: BMBWF and FWF (Austria); FNRS and FWO (Belgium); CNPq, CAPES, FAPERJ, FAPERGS, and FAPESP (Brazil); MES (Bulgaria); CERN; CAS, MoST, 
and NSFC (China); COLCIENCIAS (Colombia); MSES and CSF (Croatia); RPF (Cyprus); SENESCYT (Ecuador); MoER, ERC IUT, PUT and ERDF (Estonia); Academy of Finland, MEC, and HIP (Finland); CEA and CNRS/IN2P3 (France); BMBF, DFG, and HGF (Germany); GSRT (Greece); NKFIA (Hungary); DAE and DST (India); IPM (Iran); SFI (Ireland); INFN (Italy); MSIP and NRF (Republic of Korea); MES (Latvia); LAS (Lithuania); MOE and UM (Malaysia); BUAP, CINVESTAV, CONACYT, LNS, SEP, and UASLP-FAI (Mexico); MOS (Montenegro); MBIE (New Zealand); PAEC (Pakistan); MSHE and NSC (Poland); FCT (Portugal); JINR (Dubna); MON, RosAtom, RAS, RFBR, and NRC KI (Russia); MESTD (Serbia); SEIDI, CPAN, PCTI, and FEDER (Spain); MOSTR (Sri Lanka); Swiss Funding Agencies (Switzerland); MST (Taipei); ThEPCenter, IPST, STAR, and NSTDA (Thailand); TUBITAK and TAEK (Turkey); NASU (Ukraine); STFC (United Kingdom); DOE and NSF (USA).

[1] P. Ramond, Dual theory for free fermions, Phys. Rev. D 3, 2415 (1971).

[2] Yu. A. Gol'fand and E. P. Likhtman, Extension of the algebra of Poincare group generators and violation of $\mathrm{P}$ invariance, JETP Lett. 13, 323 (1971).

[3] S. Ferrara and B. Zumino, Supergauge invariant Yang-Mills theories, Nucl. Phys. B79, 413 (1974).

[4] J. Wess and B. Zumino, Supergauge transformations in four dimensions, Nucl. Phys. B70, 39 (1974).

[5] A. H. Chamseddine, R. Arnowitt, and P. Nath, Locally Supersymmetric Grand Unification, Phys. Rev. Lett. 49, 970 (1982).

[6] R. Barbieri, S. Ferrara, and C. A. Savoy, Gauge Models with Spontaneously Broken Local Supersymmetry, Phys. Lett. 119B, 343 (1982).

[7] L. Hall, J. Lykken, and S. Weinberg, Supergravity as the messenger of supersymmetry breaking, Phys. Rev. D 27, 2359 (1983).

[8] S. Dimopoulos and G. F. Giudice, Naturalness constraints in supersymmetric theories with nonuniversal soft terms, Phys. Lett. B 357, 573 (1995).

[9] D. N. Spergel et al. (WMAP Collaboration), First year wilkinson microwave anisotropy probe (WMAP) observations: Determination of cosmological parameters, Astrophys. J. Suppl. Ser. 148, 175 (2003).

[10] P. A. R. Ade et al. (Planck Collaboration), Planck 2015 results. XIII. Cosmological parameters, Astron. Astrophys. 594, A13 (2016).

[11] K. Griest and D. Seckel, Three exceptions in the calculation of relic abundances, Phys. Rev. D 43, 3191 (1991).

[12] R. L. Arnowitt, A. Aurisano, B. Dutta, T. Kamon, N. Korev, P. Simeon, D. A. Toback, and P. Wagner, Detection of SUSY signals in stau neutralino co-annihilation region at the LHC, AIP Conf. Proc. 903, 229 (2007).

[13] V. Khotilovich, R. L. Arnowitt, B. Dutta, and T. Kamon, The stau neutralino co-annihilation region at an international linear collider, Phys. Lett. B 618, 182 (2005).
[14] M. Carena, S. Gori, N. R. Shah, C. E. M. Wagner, and L.-T. Wang, Light stau phenomenology and the Higgs $\gamma \gamma$ rate, J. High Energy Phys. 07 (2012) 175.

[15] A. Aboubrahim, P. Nath, and A. B. Spisak, Stau coannihilation, compressed spectrum, and SUSY discovery potential at the LHC, Phys. Rev. D 95, 115030 (2017).

[16] G. H. Duan, C. Han, B. Peng, L. Wu, and J. M. Yang, Vacuum stability in stau-neutralino coannihilation in MSSM, Phys. Lett. B 788, 475 (2019).

[17] R. Arnowitt, B. Dutta, A. Gurrola, T. Kamon, A. Krislock, and D. Toback, Determining the Dark Matter Relic Density in the Minimal Supergravity Stau-Neutralino Coannihilation Region at the Large Hadron Collider, Phys. Rev. Lett. 100, 231802 (2008).

[18] B. Dutta, A. Gurrola, W. Johns, T. Kamon, P. Sheldon, and S. Kuver, Vector boson fusion processes as a probe of supersymmetric electroweak sectors at the LHC, Phys. Rev. D 87, 035029 (2013).

[19] A. G. Delannoy, B. Dutta, A. Gurrola, W. Johns, T. Kamon, E. Luiggi, A. Melo, P. Sheldon, K. Sinha, K. Wang, and S. Wu, Probing Dark Matter at the LHC Using Vector Boson Fusion Processes, Phys. Rev. Lett. 111, 061801 (2013).

[20] A. Flórez, L. Bravo, A. Gurrola, C. Ávila, M. Segura, P. Sheldon, and W. Johns, Probing the stau-neutralino coannihilation region at the LHC with a soft tau lepton and an ISR jet, Phys. Rev. D 94, 073007 (2016).

[21] B. Dutta, A. Gurrola, K. Hatakeyama, W. Johns, T. Kamon, P. Sheldon, K. Sinha, S. Wu, and Z. Wu, Probing compressed bottom squarks with boosted jets and shape analysis, Phys. Rev. D 92, 095009 (2015).

[22] B. Dutta, W. Flanagan, A. Gurrola, W. Johns, T. Kamon, P. Sheldon, K. Sinha, K. Wang, and S. Wu, Probing compressed top squarks at the LHC at $14 \mathrm{TeV}$, Phys. Rev. D 90, 095022 (2014).

[23] B. Dutta, T. Ghosh, A. Gurrola, W. Johns, T. Kamon, P. Sheldon, K. Sinha, K. Wang, and S. Wu, Probing compressed sleptons at the LHC using vector boson fusion processes, Phys. Rev. D 91, 055025 (2015).

[24] A. Flórez, A. Gurrola, W. Johns, Y. D. Oh, P. Sheldon, D. Teague, and T. Weiler, Searching for new heavy neutral gauge bosons using vector boson fusion processes at the LHC, Phys. Lett. B 767, 126 (2017).

[25] A. Flórez, K. Gui, A. Gurrola, C. Patino, and D. Restrepo, Expanding the reach of heavy neutrino searches at the LHC, Phys. Lett. B 778, 94 (2018).

[26] A. Flórez, Y. Guo, A. Gurrola, W. Johns, O. Ray, P. Sheldon, and S. Starko, Probing heavy spin-2 bosons with $\gamma \gamma$ final states from vector boson fusion processes at the LHC, Phys. Rev. D 99, 035034 (2019).

[27] CMS Collaboration, Combined search for electroweak production of charginos and neutralinos in proton-proton collisions at $\sqrt{s}=13 \mathrm{TeV}$, J. High Energy Phys. 03 (2018) 160.

[28] CMS Collaboration, Search for new physics in events with two soft oppositely charged leptons and missing transverse momentum in proton-proton collisions at $\sqrt{s}=13 \mathrm{TeV}$, Phys. Lett. B 782, 440 (2018).

[29] ATLAS Collaboration, Search for the direct production of charginos and neutralinos in final states with tau leptons in 
$\sqrt{s}=13 \mathrm{TeV} p p$ collisions with the ATLAS detector, Eur. Phys. J. C 78, 154 (2018).

[30] ATLAS Collaboration, Search for electroweak production of supersymmetric states in scenarios with compressed mass spectra at $\sqrt{s}=13 \mathrm{TeV}$ with the ATLAS detector, Phys. Rev. D 97, 052010 (2017).

[31] CMS Collaboration, Search for supersymmetry in the vector-boson fusion topology in proton-proton collisions at $\sqrt{s}=8 \mathrm{TeV}$, J. High Energy Phys. 11 (2015) 189.

[32] CMS Collaboration, Search for supersymmetry with a compressed mass spectrum in the vector boson fusion topology with 1-lepton and 0-lepton final states in proton-proton collisions at $\sqrt{s}=13 \mathrm{TeV}$, J. High Energy Phys. 08 (2019) 150.

[33] CMS Collaboration, Search for direct pair production of supersymmetric partners to the $\tau$ lepton in proton-proton collisions at $\sqrt{s}=13 \mathrm{TeV}$, arXiv:1907.13179 [Eur. Phys. J. $\mathrm{C}$ (to be published)].

[34] A. Heister et al. (ALEPH Collaboration), Search for scalar leptons in $e^{+} e^{-}$collisions at center-of-mass energies up to 209 GeV, Phys. Lett. B 526, 206 (2002).

[35] J. Abdallah et al. (DELPHI Collaboration), Searches for supersymmetric particles in $e^{+} e^{-}$collisions up to $208 \mathrm{GeV}$ and interpretation of the results within the MSSM, Eur. Phys. J. C 31, 421 (2003).

[36] P. Achard et al. (L3 Collaboration), Search for scalar leptons and scalar quarks at LEP, Phys. Lett. B 580, 37 (2004).

[37] G. Abbiendi et al. (OPAL Collaboration), Search for anomalous production of dilepton events with missing transverse momentum in $e^{+} e^{-}$collisions at $\sqrt{s}=183 \mathrm{GeV}$ to $209 \mathrm{GeV}$, Eur. Phys. J. C 32, 453 (2004).

[38] CMS Collaboration, The CMS experiment at the CERN LHC, J. Instrum. 3, S08004 (2008).

[39] CMS Collaboration, Particle-flow reconstruction and global event description with the CMS detector, J. Instrum. 12, P10003 (2017).

[40] M. Cacciari, G. P. Salam, and G. Soyez, The anti- $k_{\mathrm{T}}$ jet clustering algorithm, J. High Energy Phys. 04 (2008) 063.

[41] M. Cacciari, G. P. Salam, and G. Soyez, FastJet user manual, Eur. Phys. J. C 72, 1896 (2012).

[42] CMS Collaboration, Performance of missing transverse momentum reconstruction in proton-proton collisions at $\sqrt{s}=13 \mathrm{TeV}$ using the CMS detector, J. Instrum. 14, P07004 (2019).

[43] CMS Collaboration, Jet algorithms performance in $13 \mathrm{TeV}$ data, CMS Physics Analysis Summary Report No. CMSPAS-JME-16-003 (2016), http://cdsweb.cern.ch/record/ 2256875.

[44] CMS Collaboration, Jet energy scale and resolution in the CMS experiment in pp collisions at $8 \mathrm{TeV}$, J. Instrum. 12, P02014 (2017).

[45] CMS Collaboration, Identification of heavy-flavour jets with the CMS detector in pp collisions at $13 \mathrm{TeV}$, J. Instrum. 13, P05011 (2018).

[46] CMS Collaboration, Performance of electron reconstruction and selection with the CMS detector in proton-proton collisions at $\sqrt{s}=8 \mathrm{TeV}$, J. Instrum. 10, P06005 (2015).

[47] CMS Collaboration, Performance of the CMS muon detector and muon reconstruction with proton-proton collisions at $\sqrt{s}=13 \mathrm{TeV}$, J. Instrum. 13, P06015 (2018).
[48] CMS Collaboration, Performance of reconstruction and identification of $\tau$ leptons decaying to hadrons and $\nu_{\tau}$ in pp collisions at $\sqrt{s}=13 \mathrm{TeV}$, J. Instrum. 13, P10005 (2018).

[49] CMS Collaboration, Search for Dark Matter and Supersymmetry with a Compressed Mass Spectrum in the Vector Boson Fusion Topology in Proton-Proton Collisions at $\sqrt{s}=8 \mathrm{TeV}$, Phys. Rev. Lett. 118, 021802 (2017).

[50] A. Flórez, A. Gurrola, W. Johns, J. Maruri, P. Sheldon, K. Sinha, and S. R. Starko, Anapole dark matter via vector boson fusion processes at the LHC, Phys. Rev. D 100, 016017 (2019).

[51] J. Alwall, R. Frederix, S. Frixione, V. Hirschi, F. Maltoni, O. Mattelaer, H. S. Shao, T. Stelzer, P. Torrielli, and M. Zaro, The automated computation of tree-level and next-toleading order differential cross sections, and their matching to parton shower simulations, J. High Energy Phys. 07 (2014) 079.

[52] D. Alves (LHC New Physics Working Group), Simplified models for LHC new physics searches, J. Phys. G 39, 105005 (2012).

[53] ATLAS Collaboration, Search for the direct production of charginos, neutralinos and staus in final states with at least two hadronically decaying taus and missing transverse momentum in $p p$ collisions at $\sqrt{s}=8 \mathrm{TeV}$ with the ATLAS detector, J. High Energy Phys. 10 (2014) 96.

[54] ATLAS Collaboration, Search for the electroweak production of supersymmetric particles in $\sqrt{s}=8 \mathrm{TeV} p p$ collisions with the ATLAS detector, Phys. Rev. D 93, 052002 (2016).

[55] CMS Collaboration, Search for electroweak production of charginos in final states with two $\tau$ leptons in $p p$ collisions at $\sqrt{s}=8 \mathrm{TeV}$, J. High Energy Phys. 04 (2017) 18.

[56] A. M. Sirunyan et al., Search for supersymmetry in events with a $\tau$ lepton pair and missing transverse momentum in proton-proton collisions at $\sqrt{s}=13 \mathrm{TeV}$, J. High Energy Phys. 11 (2018) 151.

[57] ATLAS Collaboration, Search for direct stau production in events with two hadronic $\tau$-leptons in $\sqrt{s}=13 \mathrm{TeV} p p$ collisions with the ATLAS detector, arXiv:1911.06660 [Phys. Rev. D (to be published)].

[58] T. Sjöstrand, S. Ask, J. R. Christiansen, R. Corke, N. Desai, P. Ilten, S. Mrenna, S. Prestel, C. O. Rasmussen, and P.Z. Skands, An introduction to PYTHIA 8.2, Comput. Phys. Commun. 191, 159 (2015).

[59] CMS Collaboration, Event generator tunes obtained from underlying event and multiparton scattering measurements, Eur. Phys. J. C 76, 155 (2016).

[60] CMS Collaboration, Extraction and validation of a new set of CMS PYTHIA8 tunes from underlying-event measurements, Eur. Phys. J. C 80, 4 (2020).

[61] Richard D. Ball et al. (NNPDF Collaboration), Parton distributions for the LHC Run II, J. High Energy Phys. 04 (2015) 040.

[62] S. Agostinelli et al. (GEANT4 Collaboration), GEANT4A simulation toolkit, Nucl. Instrum. Methods Phys. Res., Sect. A 506, 250 (2003).

[63] CMS Collaboration, The fast simulation of the CMS detector at LHC, J. Phys. Conf. Ser. 331, 032049 (2011). 
[64] J. Debove, B. Fuks, and M. Klasen, Threshold resummation for gaugino pair production at hadron colliders, Nucl. Phys. B842, 51 (2011).

[65] B. Fuks, M. Klasen, D. R. Lamprea, and M. Rothering, Gaugino production in proton-proton collisions at a centerof-mass energy of $8 \mathrm{TeV}$, J. High Energy Phys. 10 (2012) 081.

[66] B. Fuks, M. Klasen, D. R. Lamprea, and M. Rothering, Precision predictions for electroweak superpartner production at hadron colliders with RESUMMINO, Eur. Phys. J. C 73, 2480 (2013).

[67] J. Fiaschi and M. Klasen, Neutralino-chargino pair production at NLO + NLL with resummation-improved parton density functions for LHC Run II, Phys. Rev. D 98, 055014 (2018).

[68] CMS Collaboration, The CMS trigger system, J. Instrum. 12, P01020 (2017).

[69] CMS Collaboration, Search for heavy neutrinos or thirdgeneration leptoquarks in final states with two hadronically decaying tau leptons and two jets in proton-proton collisions at $\sqrt{s}=13 \mathrm{TeV}$, J. High Energy Phys. 03 (2017) 077.

[70] CMS Collaboration, Search for heavy neutrinos and thirdgeneration leptoquarks in hadronic states of two $\tau$ leptons and two jets in proton-proton collisions at $\sqrt{s}=13 \mathrm{TeV}$, J. High Energy Phys. 03 (2019) 170.

[71] CMS Collaboration, Search for heavy resonances decaying to tau lepton pairs in proton-proton collisions at $\sqrt{s}=13 \mathrm{TeV}$, J. High Energy Phys. 02 (2017) 048.

[72] CMS Collaboration, Measurement of the inclusive $W$ and $Z$ production cross sections in pp collisions at $\sqrt{s}=7 \mathrm{TeV}$, J. High Energy Phys. 10 (2011) 132.
[73] J. Butterworth et al., PDF4LHC recommendations for LHC Run II, J. Phys. G 43, 023001 (2016).

[74] P. M. Nadolsky, H.-L. Lai, Q.-H. Cao, J. Huston, J. Pumplin, D. Stump, W.-K. Tung, and C.-P. Yuan, Implications of CTEQ global analysis for collider observables, Phys. Rev. D 78, 013004 (2008).

[75] A. D. Martin, W. J. Stirling, R. S. Thorne, and G. Watt, Update of parton distributions at NNLO, Phys. Lett. B 652, 292 (2007).

[76] M. Ubiali, NNPDF1.0 parton set for the LHC, Nucl. Phys. B, Proc. Suppl. 186, 62 (2009).

[77] CMS Collaboration, CMS luminosity measurements for the 2016 data-taking period, CMS Physics Analysis Summary Report No. CMS-PAS-LUM-17-001 (2017), http://cds.cern .ch/record/2257069.

[78] CMS Collaboration, CMS luminosity measurement for the 2017 data-taking period at $\sqrt{s}=13 \mathrm{TeV}$, CMS Physics Analysis Summary Report No. CMS-PAS-LUM-17-004 (2018), http://cds.cern.ch/record/2621960.

[79] G. Cowan, K. Cranmer, E. Gross, and O. Vitells, Asymptotic formulae for likelihood-based tests of new physics, Eur. Phys. J. C 71, 1554 (2011); Erratum, Eur. Phys. J. C 73, 2501 (2013).

[80] T. Junk, Confidence level computation for combining searches with small statistics, Nucl. Instrum. Methods Phys. Res., Sect. A 434, 435 (1999).

[81] A. L. Read, Presentation of search results: The $C L_{s}$ technique, J. Phys. G 28, 2693 (2002).

[82] R. J. Barlow and C. Beeston, Fitting using finite Monte Carlo samples, Comput. Phys. Commun. 77, 219 (1993).

A. M. Sirunyan, ${ }^{1, a}$ A. Tumasyan, ${ }^{1}$ W. Adam, ${ }^{2}$ F. Ambrogi, ${ }^{2}$ T. Bergauer, ${ }^{2}$ J. Brandstetter, ${ }^{2}$ M. Dragicevic, ${ }^{2}$ J. Erö, ${ }^{2}$ A. Escalante Del Valle, ${ }^{2}$ M. Flechl, ${ }^{2}$ R. Frühwirth, ${ }^{2, b}$ M. Jeitler, ${ }^{2, b}$ N. Krammer, ${ }^{2}$ I. Krätschmer, ${ }^{2}$ D. Liko, ${ }^{2}$ T. Madlener, ${ }^{2}$ I. Mikulec, ${ }^{2}$ N. Rad, ${ }^{2}$ J. Schieck, ${ }^{2, b}$ R. Schöfbeck, ${ }^{2}$ M. Spanring, ${ }^{2}$ D. Spitzbart, ${ }^{2}$ W. Waltenberger, ${ }^{2}$ C.-E. Wulz, ${ }^{2, b}$ M. Zarucki, ${ }^{2}$ V. Drugakov, ${ }^{3}$ V. Mossolov, ${ }^{3}$ J. Suarez Gonzalez, ${ }^{3}$ M. R. Darwish, ${ }^{4}$ E. A. De Wolf, ${ }^{4}$ D. Di Croce, ${ }^{4}$ X. Janssen, ${ }^{4}$ A. Lelek, ${ }^{4}$ M. Pieters, ${ }^{4}$ H. Rejeb Sfar, ${ }^{4}$ H. Van Haevermaet, ${ }^{4}$ P. Van Mechelen, ${ }^{4}$ S. Van Putte, ${ }^{4}$ N. Van Remortel, ${ }^{4}$ F. Blekman, ${ }^{5}$ E. S. Bols, ${ }^{5}$ S. S. Chhibra, ${ }^{5}$ J. D'Hondt, ${ }^{5}$ J. De Clercq,${ }^{5}$ D. Lontkovskyi, ${ }^{5}$ S. Lowette, ${ }^{5}$ I. Marchesini, ${ }^{5}$ S. Moortgat, ${ }^{5}$ Q. Python, ${ }^{5}$ K. Skovpen, ${ }^{5}$ S. Tavernier, ${ }^{5}$ W. Van Doninck, ${ }^{5}$ P. Van Mulders, ${ }^{5}$ D. Beghin, ${ }^{6}$ B. Bilin, ${ }^{6}$ H. Brun, ${ }^{6}$ B. Clerbaux, ${ }^{6}$ G. De Lentdecker, ${ }^{6}$ H. Delannoy, ${ }^{6}$ B. Dorney, ${ }^{6}$ L. Favart, ${ }^{6}$ A. Grebenyuk, ${ }^{6}$ A. K. Kalsi, ${ }^{6}$ A. Popov, ${ }^{6}$ N. Postiau, ${ }^{6}$ E. Starling, ${ }^{6}$ L. Thomas, ${ }^{6}$ C. Vander Velde, ${ }^{6}$ P. Vanlaer, ${ }^{6}$ D. Vannerom, ${ }^{6}$ T. Cornelis, ${ }^{7}$ D. Dobur, ${ }^{7}$ I. Khvastunov, ${ }^{7, \mathrm{c}}$ M. Niedziela, ${ }^{7}$ C. Roskas, ${ }^{7}$ M. Tytgat, ${ }^{7}$ W. Verbeke, ${ }^{7}$ B. Vermassen, ${ }^{7}$ M. Vit, ${ }^{7}$ O. Bondu, ${ }^{8}$ G. Bruno, ${ }^{8}$ C. Caputo, ${ }^{8}$ P. David, ${ }^{8}$ C. Delaere, ${ }^{8}$ M. Delcourt,${ }^{8}$ A. Giammanco, ${ }^{8}$ V. Lemaitre, ${ }^{8}$ J. Prisciandaro, ${ }^{8}$ A. Saggio, ${ }^{8}$ M. Vidal Marono, ${ }^{8}$ P. Vischia, ${ }^{8}$ J. Zobec, ${ }^{8}$ F. L. Alves, ${ }^{9}$ G. A. Alves, ${ }^{9}$ G. Correia Silva, ${ }^{9}$ C. Hensel, ${ }^{9}$ A. Moraes, ${ }^{9}$ P. Rebello Teles, ${ }^{9}$ E. Belchior Batista Das Chagas, ${ }^{10}$ W. Carvalho, ${ }^{10}$ J. Chinellato, ${ }^{10, d}$ E. Coelho, ${ }^{10}$ E. M. Da Costa, ${ }^{10}$ G. G. Da Silveira, ${ }^{10, \mathrm{e}}$ D. De Jesus Damiao, ${ }^{10}$ C. De Oliveira Martins, ${ }^{10}$ S. Fonseca De Souza, ${ }^{10}$ L. M. Huertas Guativa, ${ }^{10}$ H. Malbouisson, ${ }^{10}$ J. Martins,,${ }^{10, \mathrm{f}}$ D. Matos Figueiredo, ${ }^{10}$ M. Medina Jaime,,${ }^{10, g}$ M. Melo De Almeida, ${ }^{10}$ C. Mora Herrera, ${ }^{10}$ L. Mundim, ${ }^{10}$ H. Nogima, ${ }^{10}$ W. L. Prado Da Silva, ${ }^{10}$ L. J. Sanchez Rosas, ${ }^{10}$ A. Santoro, ${ }^{10}$ A. Sznajder ${ }^{10}$ M. Thiel, ${ }^{10}$ E. J. Tonelli Manganote, ${ }^{10, d}$ F. Torres Da Silva De Araujo, ${ }^{10}$ A. Vilela Pereira, ${ }^{10}$ C. A. Bernardes, ${ }^{11 a}$ L. Calligaris, ${ }^{11 a}$

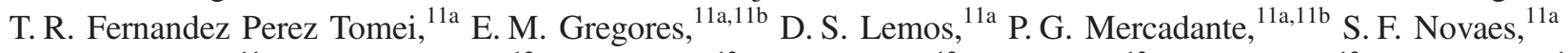
Sandra S. Padula, ${ }^{11 a}$ A. Aleksandrov, ${ }^{12}$ G. Antchev, ${ }^{12}$ R. Hadjiiska, ${ }^{12}$ P. Iaydjiev, ${ }^{12}$ M. Misheva, ${ }^{12}$ M. Rodozov, ${ }^{12}$ M. Shopova, ${ }^{12}$ G. Sultanov, ${ }^{12}$ M. Bonchev, ${ }^{13}$ A. Dimitrov, ${ }^{13}$ T. Ivanov, ${ }^{13}$ L. Litov,${ }^{13}$ B. Pavlov, ${ }^{13}$ P. Petkov, ${ }^{13}$ W. Fang, ${ }^{14, h}$ X. Gao, ${ }^{14, h}$ L. Yuan, ${ }^{14}$ G. M. Chen, ${ }^{15}$ H. S. Chen, ${ }^{15}$ M. Chen, ${ }^{15}$ C. H. Jiang, ${ }^{15}$ D. Leggat, ${ }^{15}$ H. Liao, ${ }^{15}$ Z. Liu, ${ }^{15}$ A. Spiezia, ${ }^{15}$ 
J. Tao, ${ }^{15}$ E. Yazgan, ${ }^{15}$ H. Zhang, ${ }^{15}$ S. Zhang, ${ }^{15,1}$ J. Zhao, ${ }^{15}$ A. Agapitos, ${ }^{16}$ Y. Ban, ${ }^{16}$ G. Chen, ${ }^{16}$ A. Levin, ${ }^{16}$ J. Li, ${ }^{16}$ L. Li, ${ }^{16}$ Q. Li, ${ }^{16}$ Y. Mao, ${ }^{16}$ S. J. Qian, ${ }^{16}$ D. Wang, ${ }^{16}$ Q. Wang, ${ }^{16}$ M. Ahmad,${ }^{17}$ Z. Hu, ${ }^{17}$ Y. Wang, ${ }^{17}$ M. Xiao, ${ }^{18}$ C. Avila, ${ }^{19}$ A. Cabrera, ${ }^{19}$ C. Florez, ${ }^{19}$ C. F. González Hernández, ${ }^{19}$ M. A. Segura Delgado, ${ }^{19}$ J. Mejia Guisao, ${ }^{20}$ J. D. Ruiz Alvarez, ${ }^{20}$ C. A. Salazar González, ${ }^{20}$ N. Vanegas Arbelaez, ${ }^{20}$ D. Giljanović, ${ }^{21}$ N. Godinovic, ${ }^{21}$ D. Lelas, ${ }^{21}$ I. Puljak, ${ }^{21}$ T. Sculac, ${ }^{21}$ Z. Antunovic, ${ }^{22}$ M. Kovac, ${ }^{22}$ V. Brigljevic, ${ }^{23}$ D. Ferencek, ${ }^{23}$ K. Kadija, ${ }^{23}$ B. Mesic, ${ }^{23}$ M. Roguljic, ${ }^{23}$ A. Starodumov, ${ }^{23, j}$ T. Susa, ${ }^{23}$ M. W. Ather ${ }^{24}$ A. Attikis, ${ }^{24}$ E. Erodotou, ${ }^{24}$ A. Ioannou, ${ }^{24}$ M. Kolosova, ${ }^{24}$ S. Konstantinou, ${ }^{24}$

G. Mavromanolakis,${ }^{24}$ J. Mousa, ${ }^{24}$ C. Nicolaou, ${ }^{24}$ F. Ptochos,${ }^{24}$ P. A. Razis, ${ }^{24}$ H. Rykaczewski, ${ }^{24}$ D. Tsiakkouri, ${ }^{24}$ M. Finger, ${ }^{25, \mathrm{k}}$ M. Finger Jr., ${ }^{25, \mathrm{k}}$ A. Kveton, ${ }^{25}$ J. Tomsa, ${ }^{25}$ E. Ayala, ${ }^{26}$ E. Carrera Jarrin, ${ }^{27}$ Y. Assran, ${ }^{28,1, \mathrm{~m}}$ S. Elgammal, ${ }^{28,1}$ S. Bhowmik, ${ }^{29}$ A. Carvalho Antunes De Oliveira, ${ }^{29}$ R. K. Dewanjee, ${ }^{29}$ K. Ehataht, ${ }^{29}$ M. Kadastik,${ }^{29}$ M. Raidal, ${ }^{29}$ C. Veelken, ${ }^{29}$ P. Eerola, ${ }^{30}$ L. Forthomme, ${ }^{30}$ H. Kirschenmann, ${ }^{30}$ K. Osterberg,${ }^{30}$ M. Voutilainen, ${ }^{30}$ F. Garcia, ${ }^{31}$

J. Havukainen, ${ }^{31}$ J. K. Heikkilä, ${ }^{31}$ V. Karimäki, ${ }^{31}$ M. S. Kim, ${ }^{31}$ R. Kinnunen, ${ }^{31}$ T. Lampén, ${ }^{31}$ K. Lassila-Perini, ${ }^{31}$ S. Laurila, ${ }^{31}$ S. Lehti ${ }^{31}$ T. Lindén, ${ }^{31}$ P. Luukka, ${ }^{31}$ T. Mäenpää, ${ }^{31}$ H. Siikonen, ${ }^{31}$ E. Tuominen, ${ }^{31}$ J. Tuominiemi, ${ }^{31}$ T. Tuuva, ${ }^{32}$ M. Besancon, ${ }^{33}$ F. Couderc, ${ }^{33}$ M. Dejardin, ${ }^{33}$ D. Denegri, ${ }^{33}$ B. Fabbro, ${ }^{33}$ J. L. Faure, ${ }^{33}$ F. Ferri, ${ }^{33}$ S. Ganjour, ${ }^{33}$ A. Givernaud, ${ }^{33}$ P. Gras, ${ }^{33}$ G. Hamel de Monchenault, ${ }^{33}$ P. Jarry,${ }^{33}$ C. Leloup, ${ }^{33}$ B. Lenzi, ${ }^{33}$ E. Locci, ${ }^{33}$ J. Malcles, ${ }^{33}$ J. Rander, ${ }^{33}$ A. Rosowsky, ${ }^{33}$ M. Ö. Sahin, ${ }^{33}$ A. Savoy-Navarro, ${ }^{33, n}$ M. Titov,${ }^{33}$ G. B. Yu, ${ }^{33}$ S. Ahuja, ${ }^{34}$ C. Amendola, ${ }^{34}$ F. Beaudette, ${ }^{34}$ P. Busson, ${ }^{34}$ C. Charlot,${ }^{34}$ B. Diab,${ }^{34}$ G. Falmagne, ${ }^{34}$ R. Granier de Cassagnac, ${ }^{34}$ I. Kucher, ${ }^{34}$ A. Lobanov,${ }^{34}$ C. Martin Perez,${ }^{34}$ M. Nguyen, ${ }^{34}$ C. Ochando, ${ }^{34}$ P. Paganini, ${ }^{34}$ J. Rembser, ${ }^{34}$ R. Salerno, ${ }^{34}$ J. B. Sauvan, ${ }^{34}$ Y. Sirois, ${ }^{34}$ A. Zabi, ${ }^{34}$ A. Zghiche, ${ }^{34}$ J.-L. Agram, ${ }^{35,0}$ J. Andrea, ${ }^{35}$ D. Bloch, ${ }^{35}$ G. Bourgatte, ${ }^{35}$ J.-M. Brom, ${ }^{35}$ E. C. Chabert, ${ }^{35}$ C. Collard, ${ }^{35}$ E. Conte, ${ }^{35,0}$ J.-C. Fontaine,${ }^{35,0}$ D. Gelé, ${ }^{35}$ U. Goerlach, ${ }^{35}$ M. Jansová, ${ }^{35}$ A.-C. Le Bihan, ${ }^{35}$ N. Tonon, ${ }^{35}$ P. Van Hove, ${ }^{35}$ S. Gadrat,${ }^{36}$ S. Beauceron, ${ }^{37}$ C. Bernet,${ }^{37}$ G. Boudoul,${ }^{37}$ C. Camen,${ }^{37}$ A. Carle,${ }^{37}$ N. Chanon, ${ }^{37}$ R. Chierici, ${ }^{37}$ D. Contardo,${ }^{37}$ P. Depasse, ${ }^{37}$ H. El Mamouni, ${ }^{37}$ J. Fay, ${ }^{37}$ S. Gascon, ${ }^{37}$ M. Gouzevitch, ${ }^{37}$ B. Ille, ${ }^{37}$ Sa. Jain, ${ }^{37}$ F. Lagarde, ${ }^{37}$ I. B. Laktineh, ${ }^{37}$ H. Lattaud, ${ }^{37}$ A. Lesauvage, ${ }^{37}$ M. Lethuillier,${ }^{37}$ L. Mirabito, ${ }^{37}$ S. Perries,${ }^{37}$ V. Sordini, ${ }^{37}$ L. Torterotot,${ }^{37}$

G. Touquet, ${ }^{37}$ M. Vander Donckt, ${ }^{37}$ S. Viret, ${ }^{37}$ A. Khvedelidze, ${ }^{38, k}$ Z. Tsamalaidze, ${ }^{39, k}$ C. Autermann, ${ }^{40}$ L. Feld, ${ }^{40}$ M. K. Kiesel, ${ }^{40}$ K. Klein, ${ }^{40}$ M. Lipinski, ${ }^{40}$ D. Meuser, ${ }^{40}$ A. Pauls,${ }^{40}$ M. Preuten, ${ }^{40}$ M. P. Rauch, ${ }^{40}$ J. Schulz,${ }^{40}$ M. Teroerde, ${ }^{40}$ B. Wittmer, ${ }^{40}$ M. Erdmann, ${ }^{41}$ B. Fischer, ${ }^{41}$ S. Ghosh, ${ }^{41}$ T. Hebbeker, ${ }^{41}$ K. Hoepfner ${ }^{41}$ H. Keller, ${ }^{41}$ L. Mastrolorenzo, ${ }^{41}$ M. Merschmeyer, ${ }^{41}$ A. Meyer, ${ }^{41}$ P. Millet,${ }^{41}$ G. Mocellin, ${ }^{41}$ S. Mondal,${ }^{41}$ S. Mukherjee, ${ }^{41}$ D. Noll, ${ }^{41}$ A. Novak, ${ }^{41}$ T. Pook, ${ }^{41}$ A. Pozdnyakov, ${ }^{41}$ T. Quast,${ }^{41}$ M. Radziej, ${ }^{41}$ Y. Rath, ${ }^{41}$ H. Reithler, ${ }^{41}$ J. Roemer, ${ }^{41}$ A. Schmidt, ${ }^{41}$ S. C. Schuler, ${ }^{41}$ A. Sharma, ${ }^{41}$ S. Wiedenbeck, ${ }^{41}$ S. Zaleski, ${ }^{41}$ G. Flügge, ${ }^{42}$ W. Haj Ahmad ${ }^{42, p}$ O. Hlushchenko, ${ }^{42}$ T. Kress, ${ }^{42}$ T. Müller ${ }^{42}$ A. Nowack, ${ }^{42}$ C. Pistone, ${ }^{42}$ O. Pooth, ${ }^{42}$ D. Roy, ${ }^{42}$ H. Sert ${ }^{42}$ A. Stahl ${ }^{42, q}$ M. Aldaya Martin, ${ }^{43}$ P. Asmuss, ${ }^{43}$ I. Babounikau, ${ }^{43}$ H. Bakhshiansohi, ${ }^{43}$ K. Beernaert, ${ }^{43}$ O. Behnke, ${ }^{43}$ A. Bermúdez Martínez, ${ }^{43}$ D. Bertsche, ${ }^{43}$ A. A. Bin Anuar, ${ }^{43}$ K. Borras,${ }^{43, r}$ V. Botta, ${ }^{43}$ A. Campbell, ${ }^{43}$ A. Cardini,${ }^{43}$ P. Connor, ${ }^{43}$ S. Consuegra Rodríguez, ${ }^{43}$ C. Contreras-Campana,${ }^{43}$ V. Danilov, ${ }^{43}$ A. De Wit, ${ }^{43}$ M. M. Defranchis, ${ }^{43}$ C. Diez Pardos,${ }^{43}$ D. Domínguez Damiani, ${ }^{43}$ G. Eckerlin, ${ }^{43}$ D. Eckstein, ${ }^{43}$ T. Eichhorn, ${ }^{43}$ A. Elwood, ${ }^{43}$ E. Eren, ${ }^{43}$ E. Gallo, ${ }^{43,}$ A. Geiser, ${ }^{43}$ A. Grohsjean, ${ }^{43}$ M. Guthoff, ${ }^{43}$ M. Haranko, ${ }^{43}$ A. Harb ${ }^{43}$ A. Jafari ${ }^{43}$ N. Z. Jomhari, ${ }^{43}$ H. Jung, ${ }^{43}$ A. Kasem, ${ }^{43, r}$ M. Kasemann, ${ }^{43}$ H. Kaveh, ${ }^{43}$ J. Keaveney, ${ }^{43}$ C. Kleinwort, ${ }^{43}$ J. Knolle, ${ }^{43}$ D. Krücker, ${ }^{43}$ W. Lange, ${ }^{43}$ T. Lenz, ${ }^{43}$ J. Lidrych,${ }^{43}$ K. Lipka, ${ }^{43}$ W. Lohmann, ${ }^{43, t}$ R. Mankel,${ }^{43}$ I.-A. Melzer-Pellmann, ${ }^{43}$ A. B. Meyer, ${ }^{43}$ M. Meyer, ${ }^{43}$ M. Missiroli, ${ }^{43}$ G. Mittag, ${ }^{43}$ J. Mnich, ${ }^{43}$ A. Mussgiller, ${ }^{43}$ V. Myronenko, ${ }^{43}$ D. Pérez Adán, ${ }^{43}$ S. K. Pflitsch, ${ }^{43}$ D. Pitzl, ${ }^{43}$ A. Raspereza, ${ }^{43}$ A. Saibel,${ }^{43}$ M. Savitskyi,${ }^{43}$ V. Scheurer, ${ }^{43}$ P. Schütze,${ }^{43}$ C. Schwanenberger,${ }^{43}$ R. Shevchenko, ${ }^{43}$ A. Singh, ${ }^{43}$ H. Tholen, ${ }^{43}$ O. Turkot ${ }^{43}$ A. Vagnerini, ${ }^{43}$ M. Van De Klundert,${ }^{43}$ R. Walsh, ${ }^{43}$ Y. Wen, ${ }^{43}$ K. Wichmann, ${ }^{43}$ C. Wissing, ${ }^{43}$ O. Zenaiev, ${ }^{43}$ R. Zlebcik, ${ }^{43}$ R. Aggleton, ${ }^{44}$ S. Bein, ${ }^{44}$ L. Benato, ${ }^{44}$ A. Benecke, ${ }^{44}$ V. Blobel, ${ }^{44}$ T. Dreyer, ${ }^{44}$ A. Ebrahimi, ${ }^{44}$ F. Feindt, ${ }^{44}$ A. Fröhlich, ${ }^{44}$ C. Garbers,${ }^{44}$ E. Garutti, ${ }^{44}$ D. Gonzalez, ${ }^{44}$ P. Gunnellini, ${ }^{44}$ J. Haller,${ }^{44}$ A. Hinzmann, ${ }^{44}$ A. Karavdina, ${ }^{44}$ G. Kasieczka, ${ }^{44}$ R. Klanner, ${ }^{44}$ R. Kogler, ${ }^{44}$ N. Kovalchuk, ${ }^{44}$ S. Kurz, ${ }^{44}$ V. Kutzner, ${ }^{44}$ J. Lange, ${ }^{44}$ T. Lange, ${ }^{44}$ A. Malara, ${ }^{44}$ J. Multhaup, ${ }^{44}$ C. E. N. Niemeyer, ${ }^{44}$ A. Perieanu, ${ }^{44}$ A. Reimers ${ }^{44}$ O. Rieger ${ }^{44}$ C. Scharf, ${ }^{44}$ P. Schleper, ${ }^{44}$ S. Schumann, ${ }^{44}$ J. Schwandt, ${ }^{44}$ J. Sonneveld, ${ }^{44}$ H. Stadie ${ }^{44}$ G. Steinbrück, ${ }^{44}$ F. M. Stober, ${ }^{44}$ B. Vormwald, ${ }^{44}$ I. Zoi, ${ }^{44}$ M. Akbiyik,${ }^{45}$ C. Barth,${ }^{45}$ M. Baselga, ${ }^{45}$ S. Baur, ${ }^{45}$ T. Berger ${ }^{45}$ E. Butz, ${ }^{45}$ R. Caspart, ${ }^{45}$ T. Chwalek, ${ }^{45}$ W. De Boer, ${ }^{45}$ A. Dierlamm, ${ }^{45}$ K. El Morabit, ${ }^{45}$ N. Faltermann,${ }^{45}$ M. Giffels, ${ }^{45}$ P. Goldenzweig ${ }^{45}$ A. Gottmann, ${ }^{45}$ M. A. Harrendorf, ${ }^{45}$ F. Hartmann, ${ }^{45, q}$ U. Husemann, ${ }^{45}$ S. Kudella, ${ }^{45}$ S. Mitra,${ }^{45}$ M. U. Mozer, ${ }^{45}$ D. Müller,${ }^{45}$ Th. Müller ${ }^{45}$ M. Musich, ${ }^{45}$ A. Nürnberg, ${ }^{45}$ G. Quast,${ }^{45}$ K. Rabbertz ${ }^{45}$ M. Schröder ${ }^{45}$ I. Shvetsov, ${ }^{45}$ H. J. Simonis, ${ }^{45}$ R. Ulrich, ${ }^{45}$ M. Wassmer, ${ }^{45}$ M. Weber, ${ }^{45}$ C. Wöhrmann, ${ }^{45}$ R. Wolf, ${ }^{45}$ G. Anagnostou, ${ }^{46}$ P. Asenov, ${ }^{46}$ G. Daskalakis ${ }^{46}$ T. Geralis, ${ }^{46}$ A. Kyriakis, ${ }^{46}$ D. Loukas, ${ }^{46}$ G. Paspalaki, ${ }^{46}$ M. Diamantopoulou, ${ }^{47}$ G. Karathanasis, ${ }^{47}$ P. Kontaxakis, ${ }^{47}$ 
A. Manousakis-katsikakis, ${ }^{47}$ A. Panagiotou, ${ }^{47}$ I. Papavergou, ${ }^{47}$ N. Saoulidou, ${ }^{47}$ A. Stakia ${ }^{47}$ K. Theofilatos ${ }^{47}$ K. Vellidis,${ }^{47}$ E. Vourliotis ${ }^{47}$ G. Bakas, ${ }^{48}$ K. Kousouris,${ }^{48}$ I. Papakrivopoulos, ${ }^{48}$ G. Tsipolitis, ${ }^{48}$ I. Evangelou, ${ }^{49}$ C. Foudas, ${ }^{49}$ P. Gianneios, ${ }^{49}$ P. Katsoulis, ${ }^{49}$ P. Kokkas, ${ }^{49}$ S. Mallios, ${ }^{49}$ K. Manitara, ${ }^{49}$ N. Manthos, ${ }^{49}$ I. Papadopoulos, ${ }^{49}$ J. Strologas,${ }^{49}$ F. A. Triantis, ${ }^{49}$ D. Tsitsonis, ${ }^{49}$ M. Bartók ${ }^{50, u}$ R. Chudasama,${ }^{50}$ M. Csanad,${ }^{50}$ P. Major ${ }^{50}$ K. Mandal, ${ }^{50}$ A. Mehta, ${ }^{50}$ M. I. Nagy, ${ }^{50}$ G. Pasztor,${ }^{50}$ O. Surányi,${ }^{50}$ G. I. Veres, ${ }^{50}$ G. Bencze, ${ }^{51}$ C. Hajdu ${ }^{51}$ D. Horvath,${ }^{51, v}$ F. Sikler, ${ }^{51}$ T. Á. Vámi, ${ }^{51}$ V. Veszpremi ${ }^{51}$ G. Vesztergombi, ${ }^{51, a, w}$ N. Beni, ${ }^{52}$ S. Czellar, ${ }^{52}$ J. Karancsi,${ }^{52, u}$ A. Makovec, ${ }^{52}$ J. Molnar, ${ }^{52}$ Z. Szillasi, ${ }^{52}$ P. Raics, ${ }^{53}$ D. Teyssier, ${ }^{53}$ Z. L. Trocsanyi,${ }^{53}$ B. Ujvari, ${ }^{53}$ T. Csorgo,${ }^{54}$ W. J. Metzger, ${ }^{54}$ F. Nemes,${ }^{54}$ T. Novak, ${ }^{54}$ S. Choudhury, ${ }^{55}$ J. R. Komaragiri, ${ }^{55}$ P. C. Tiwari, ${ }^{55}$ S. Bahinipati, ${ }^{56, x}$ C. Kar, ${ }^{56}$ G. Kole, ${ }^{56}$ P. Mal, ${ }^{56}$

V. K. Muraleedharan Nair Bindhu, ${ }^{56}$ A. Nayak, ${ }^{56, y}$ D. K. Sahoo, ${ }^{56, x}$ S. K. Swain, ${ }^{56}$ S. Bansal,${ }^{57}$ S. B. Beri, ${ }^{57}$ V. Bhatnagar,${ }^{57}$ S. Chauhan, ${ }^{57}$ R. Chawla, ${ }^{57}$ N. Dhingra, ${ }^{57}$ R. Gupta, ${ }^{57}$ A. Kaur,${ }^{57}$ M. Kaur, ${ }^{57}$ S. Kaur, ${ }^{57}$ P. Kumari, ${ }^{57}$ M. Lohan, ${ }^{57}$ M. Meena ${ }^{57}$ K. Sandeep, ${ }^{57}$ S. Sharma,${ }^{57}$ J. B. Singh, ${ }^{57}$ A. K. Virdi, ${ }^{57}$ A. Bhardwaj, ${ }^{58}$ B. C. Choudhary, ${ }^{58}$ R. B. Garg, ${ }^{58}$ M. Gola ${ }^{58}$ S. Keshri, ${ }^{58}$ Ashok Kumar, ${ }^{58}$ M. Naimuddin, ${ }^{58}$ P. Priyanka, ${ }^{58}$ K. Ranjan, ${ }^{58}$ Aashaq Shah,${ }^{58}$ R. Sharma, ${ }^{58}$ R. Bhardwaj, ${ }^{59, z}$ M. Bharti, ${ }^{59, z}$ R. Bhattacharya, ${ }^{59}$ S. Bhattacharya, ${ }^{59}$ U. Bhawandeep, ${ }^{59, z}$ D. Bhowmik, ${ }^{59}$ S. Dutta, ${ }^{59}$ S. Ghosh, ${ }^{59}$ B. Gomber, ${ }^{59, \text { aa }}$ M. Maity, ${ }^{59, b b}$ K. Mondal, ${ }^{59}$ S. Nandan, ${ }^{59}$ A. Purohit, ${ }^{59}$ P. K. Rout,${ }^{59}$ G. Saha, ${ }^{59}$ S. Sarkar,${ }^{59}$ T. Sarkar, ${ }^{59, b b}$ M. Sharan, ${ }^{59}$ B. Singh, ${ }^{59, z}$ S. Thakur, ${ }^{59, z}$ P. K. Behera, ${ }^{60}$ P. Kalbhor, ${ }^{60}$ A. Muhammad, ${ }^{60}$ P. R. Pujahari, ${ }^{60}$ A. Sharma, ${ }^{60}$ A. K. Sikdar, ${ }^{60}$ D. Dutta,${ }^{61}$ V. Jha, ${ }^{61}$ V. Kumar, ${ }^{61}$ D. K. Mishra, ${ }^{61}$ P. K. Netrakanti, ${ }^{61}$ L. M. Pant, ${ }^{61}$ P. Shukla, ${ }^{61}$ T. Aziz ${ }^{62}$ M. A. Bhat, ${ }^{62}$ S. Dugad, ${ }^{62}$ G. B. Mohanty, ${ }^{62}$ N. Sur, ${ }^{62}$ Ravindra Kumar Verma, ${ }^{62}$ S. Banerjee, ${ }^{63}$ S. Bhattacharyaa ${ }^{63}$ S. Chatterjee, ${ }^{63}$ P. Das, ${ }^{63}$ M. Guchait, ${ }^{63}$ S. Karmakar, ${ }^{63}$ S. Kumar, ${ }^{63}$ G. Majumder, ${ }^{63}$ K. Mazumdar,${ }^{63}$ N. Sahoo, ${ }^{63}$ S. Sawant, ${ }^{63}$ S. Dube, ${ }^{64}$ B. Kansal,${ }^{64}$ A. Kapoor, ${ }^{64}$ K. Kothekar,${ }^{64}$ S. Pandey,${ }^{64}$ A. Rane,${ }^{64}$ A. Rastogi,${ }^{64}$ S. Sharma, ${ }^{64}$ S. Chenarani, ${ }^{65, \mathrm{cc}}$ E. Eskandari Tadavani, ${ }^{65}$ S. M. Etesami,${ }^{65, \mathrm{cc}}$ M. Khakzad, ${ }^{65}$ M. Mohammadi Najafabadi, ${ }^{65}$ M. Naseri, ${ }^{65}$ F. Rezaei Hosseinabadi, ${ }^{65}$ M. Felcini ${ }^{66}$ M. Grunewald, ${ }^{66}$ M. Abbrescia,${ }^{67,67 b}$ R. Aly, ${ }^{67 a, 67 b, d d}$ C. Calabria, ${ }^{67 a, 67 b}$ A. Colaleo, ${ }^{67 \mathrm{a}}$ D. Creanza, ${ }^{67 a, 67 \mathrm{c}}$ L. Cristella,${ }^{67 a, 67 \mathrm{~b}}$ N. De Filippis,${ }^{67 a, 67 \mathrm{c}}$ M. De Palma, ${ }^{67,67 \mathrm{~b}}$ A. Di Florio, ${ }^{67 a, 67 b}$ W. Elmetenawee, ${ }^{67 a, 67 b}$ L. Fiore, ${ }^{67 a}$ A. Gelmi, ${ }^{67 a, 67 b}$ G. Iaselli, ${ }^{67 a, 67 c}$ M. Ince,${ }^{67 a, 67 b}$ S. Lezki, ${ }^{67 a, 67 b}$ G. Maggi, ${ }^{67 a, 67 c}$ M. Maggi, ${ }^{67 \mathrm{a}}$ G. Miniello ${ }^{67 \mathrm{a}, 67 \mathrm{~b}}$ S. My, ${ }^{67 \mathrm{a}, 67 \mathrm{~b}}$ S. Nuzzo, ${ }^{67,67 \mathrm{~b}}$ A. Pompili, ${ }^{67,67 \mathrm{~b}}$ G. Pugliese, ${ }^{67 a, 67 \mathrm{c}}$ R. Radogna, ${ }^{67 \mathrm{a}}$ A. Ranieri, ${ }^{67 a}$ G. Selvaggi, ${ }^{67,67 b}$ L. Silvestris, ${ }^{67 a}$ F. M. Simone, ${ }^{67 a, 67 b}$ R. Venditti, ${ }^{67 a}$ P. Verwilligen, ${ }^{67 a}$ G. Abbiendi, ${ }^{68 a}$ C. Battilana, ${ }^{68 a, 68 b}$ D. Bonacorsi, ${ }^{68 a, 68 b}$ L. Borgonovi, ${ }^{68 a, 68 b}$ S. Braibant-Giacomelli, ${ }^{68 a, 68 b}$ R. Campanini, ${ }^{68 a, 68 b}$

P. Capiluppi ${ }^{68 a, 68 b}$ A. Castro, ${ }^{68 a, 68 b}$ F. R. Cavallo, ${ }^{68 \mathrm{a}}$ C. Ciocca,${ }^{68 \mathrm{a}}$ G. Codispoti, ${ }^{68 \mathrm{a}, 68 \mathrm{~b}}$ M. Cuffiani, ${ }^{68 \mathrm{a}, 68 \mathrm{~b}}$ G. M. Dallavalle, ${ }^{68 \mathrm{a}}$

F. Fabbri, ${ }^{68 \mathrm{a}}$ A. Fanfani, ${ }^{68 \mathrm{a}, 68 \mathrm{~b}}$ E. Fontanesi, ${ }^{68 \mathrm{a}, 68 \mathrm{~b}}$ P. Giacomelli, ${ }^{68 \mathrm{a}}$ C. Grandi, ${ }^{68 \mathrm{a}}$ L. Guiducci, ${ }^{6 \mathrm{a}, 68 \mathrm{~b}}$ F. Iemmi, ${ }^{68 a, 68 \mathrm{~b}}$ S. Lo Meo, ${ }^{68 a, e e}$ S. Marcellini, ${ }^{68 \mathrm{a}}$ G. Masetti, ${ }^{68 \mathrm{a}}$ F. L. Navarria ${ }^{68 \mathrm{a}, 68 \mathrm{~b}}$ A. Perrotta, ${ }^{68 \mathrm{a}}$ F. Primavera, ${ }^{68 \mathrm{a}, 68 \mathrm{~b}}$ A. M. Rossi, ${ }^{68 \mathrm{a}, 68 \mathrm{~b}}$ T. Rovelli, ${ }^{68 a, 68 b}$ G. P. Siroli, ${ }^{68 a, 68 b}$ N. Tosi, ${ }^{68 a}$ S. Albergo, ${ }^{69 a, 69 b, f f}$ S. Costa, ${ }^{69 a, 69 b}$ A. Di Mattia, ${ }^{69 a}$ R. Potenza, ${ }^{69 a, 69 b}$

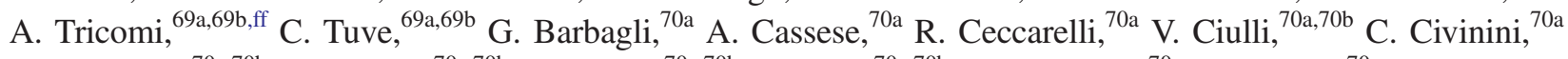
R. D’Alessandro, ${ }^{70 a, 70 b}$ E. Focardi, ${ }^{70 a, 70 b}$ G. Latino, ${ }^{70 a, 70 b}$ P. Lenzi, ${ }^{70 a, 70 b}$ M. Meschini, ${ }^{70 a}$ S. Paoletti, ${ }^{70 a}$ G. Sguazzoni, ${ }^{70 a}$ L. Viliani ${ }^{70 a}$ L. Benussi, ${ }^{71}$ S. Bianco, ${ }^{71}$ D. Piccolo, ${ }^{71}$ M. Bozzo, ${ }^{72 a, 72 b}$ F. Ferro, ${ }^{72 a}$ R. Mulargia, ${ }^{72 a, 72 b}$ E. Robutti, ${ }^{72 a}$ S. Tosi,${ }^{72 a, 72 b}$ A. Benaglia, ${ }^{73 a}$ A. Beschi,${ }^{73 a, 73 b}$ F. Brivio, ${ }^{73 a, 73 b}$ V. Ciriolo, ${ }^{73 a, 73 b, q}$ S. Di Guida, ${ }^{73 a, 73 b, q}$ M. E. Dinardo, ${ }^{73 a, 73 b}$ P. Dini, ${ }^{73 a}$ S. Gennai, ${ }^{73 a}$ A. Ghezzi, ${ }^{73 a, 73 b}$ P. Govoni, ${ }^{73 a, 73 b}$ L. Guzzi, ${ }^{73 a, 73 b}$ M. Malberti, ${ }^{73 a}$ S. Malvezzi, ${ }^{73 a}$ D. Menasce, ${ }^{73 a}$ F. Monti, ${ }^{73 a, 73 b}$ L. Moroni, ${ }^{73 a}$ M. Paganoni, ${ }^{73 a, 73 b}$ D. Pedrini, ${ }^{73 a}$ S. Ragazzi, ${ }^{73 a, 73 b}$ T. Tabarelli de Fatis, ${ }^{73 a, 73 b}$ D. Zuolo, ${ }^{73 a, 73 b}$ S. Buontempo, ${ }^{74 \mathrm{a}}$ N. Cavallo, ${ }^{74 a, 74 \mathrm{c}}$ A. De Iorio ${ }^{74 \mathrm{a}, 74 \mathrm{~b}}$ A. Di Crescenzo, ${ }^{74 \mathrm{a}, 74 \mathrm{~b}}$ F. Fabozzi, ${ }^{74 a, 74 \mathrm{c}}$ F. Fienga, ${ }^{74 \mathrm{a}}$ G. Galati, ${ }^{74 \mathrm{a}}$ A. O. M. Iorio, ${ }^{74 a, 74 b}$ L. Lista, ${ }^{74 a, 74 b}$ S. Meola, ${ }^{74 a, 74 d, q}$ P. Paolucci, ${ }^{74, q}$ B. Rossi ${ }^{74 a}$ C. Sciacca, ${ }^{74 a, 74 b}$ E. Voevodina,${ }^{74 a, 74 b}$ P. Azzi ${ }^{75 a}$ N. Bacchetta, ${ }^{75 a}$ D. Bisello, ${ }^{75 a, 75 b}$ A. Boletti ${ }^{75 a, 75 b}$ A. Bragagnolo, ${ }^{75 a, 75 b}$ R. Carlin, ${ }^{75 a, 75 b}$ P. Checchia, ${ }^{75 a}$ P. De Castro Manzano, ${ }^{75 a}$ T. Dorigo ${ }^{75 a}$ U. Dosselli, ${ }^{75 a}$ F. Gasparini, ${ }^{75 a, 75 b}$ U. Gasparini, ${ }^{75 a, 75 b}$ A. Gozzelino, ${ }^{75 a}$ S. Y. Hoh, ${ }^{75 a, 75 b}$ P. Lujan, ${ }^{75 a}$ M. Margoni, ${ }^{75 a, 75 b}$ A. T. Meneguzzo, ${ }^{75 a, 75 b}$ J. Pazzini, ${ }^{75 a, 75 b}$ M. Presilla ${ }^{75 a, 75 b}$ P. Ronchese, ${ }^{75 a, 75 b}$ R. Rossin, ${ }^{75 a, 75 b}$ F. Simonetto, ${ }^{75 a, 75 b}$ A. Tiko, ${ }^{75 a}$ M. Tosi, ${ }^{75 a, 75 b}$ M. Zanetti, ${ }^{75 a, 75 b}$ P. Zotto, ${ }^{75 a, 75 b}$ G. Zumerle, ${ }^{75 a, 75 b}$ A. Braghieri, ${ }^{76 \mathrm{a}}$ D. Fiorina, ${ }^{76 a, 76 \mathrm{~b}}$ P. Montagna ${ }^{76 \mathrm{a}, 76 \mathrm{~b}}$ S. P. Ratti, ${ }^{76 a, 76 \mathrm{~b}}$ V. Re,${ }^{76 \mathrm{a}}$ M. Ressegotti, ${ }^{76 a, 76 \mathrm{~b}}$ C. Riccardi, ${ }^{76 a, 76 \mathrm{~b}}$ P. Salvini, ${ }^{76 a}$ I. Vai, ${ }^{76 a}$ P. Vitulo, ${ }^{76 a, 76 b}$ M. Biasini, ${ }^{77 a, 77 b}$ G. M. Bilei, ${ }^{77 a}$ D. Ciangottini, ${ }^{77 a, 77 b}$ L. Fanò, ${ }^{77 a, 77 b}$ P. Lariccia, ${ }^{77 a, 77 b}$ R. Leonardi, ${ }^{77,77 b}$ E. Manoni, ${ }^{77 a}$ G. Mantovani, ${ }^{77,77 b}$ V. Mariani, ${ }^{77,77 b}$ M. Menichelli, ${ }^{77 a}$ A. Rossi, ${ }^{77,77 b}$

A. Santocchia, ${ }^{77 a, 77 b}$ D. Spiga, ${ }^{77 a}$ K. Androsov, ${ }^{78 a}$ P. Azzurri, ${ }^{78 a}$ G. Bagliesi, ${ }^{78 a}$ V. Bertacchi, ${ }^{78 a, 78 c}$ L. Bianchini, ${ }^{78 a}$ T. Boccali, ${ }^{78 a}$ R. Castaldi, ${ }^{78 a}$ M. A. Ciocci, ${ }^{78 a, 78 b}$ R. Dell'Orso, ${ }^{78 a}$ S. Donato, ${ }^{78 a}$ G. Fedi, ${ }^{78 a}$ L. Giannini ${ }^{78 a, 78 c}$ A. Giassi, ${ }^{78 a}$ M. T. Grippo, ${ }^{78 a}$ F. Ligabue, ${ }^{78 a, 78 c}$ E. Manca, ${ }^{78 a, 78 c}$ G. Mandorli, ${ }^{78 a, 78 c}$ A. Messineo ${ }^{78 a, 78 b}$ F. Palla, ${ }^{78 a}$ A. Rizzi, ${ }^{78 a, 78 b}$ G. Rolandi, ${ }^{78 a, g g}$ S. Roy Chowdhury, ${ }^{78 a}$ A. Scribano, ${ }^{78 a}$ P. Spagnolo, ${ }^{78 a}$ R. Tenchini, ${ }^{78 a}$ G. Tonelli, ${ }^{78 a, 78 b}$ N. Turini, ${ }^{78 a}$ 
A. Venturi, ${ }^{78 a}$ P. G. Verdini ${ }^{78 a}$ F. Cavallari ${ }^{79 a}$ M. Cipriani, ${ }^{79 a, 79 b}$ D. Del Re, ${ }^{79 a, 79 b}$ E. Di Marco, ${ }^{79 a, 79 b}$ M. Diemoz ${ }^{79 a}$

E. Longo, ${ }^{79 a, 79 b}$ P. Meridiani, ${ }^{79 a}$ G. Organtini, ${ }^{79 a, 79 b}$ F. Pandolfi, ${ }^{79 a}$ R. Paramatti, ${ }^{79 a, 79 b}$ C. Quaranta, ${ }^{79 a, 79 b}$ S. Rahatlou, ${ }^{79 a, 79 b}$

C. Rovelli, ${ }^{79 a}$ F. Santanastasio, ${ }^{79 a, 79 b}$ L. Soffi, ${ }^{79 a, 79 b}$ N. Amapane, ${ }^{80 a, 80 b}$ R. Arcidiacono, ${ }^{80 a, 80 c}$ S. Argiro, ${ }^{80 a, 80 b}$

M. Arneodo, ${ }^{80 a, 80 \mathrm{c}}$ N. Bartosik, ${ }^{80 \mathrm{a}}$ R. Bellan, ${ }^{80 \mathrm{a}, 80 \mathrm{~b}}$ A. Bellora ${ }^{80 \mathrm{a}}$ C. Biino, ${ }^{80 \mathrm{a}}$ A. Cappati,${ }^{80 \mathrm{a}, 80 \mathrm{~b}}$ N. Cartiglia,${ }^{80 \mathrm{a}}$ S. Cometti, ${ }^{80 \mathrm{a}}$ M. Costa, ${ }^{80 a, 80 b}$ R. Covarelli, ${ }^{80 a, 80 b}$ N. Demaria, ${ }^{80 a}$ B. Kiani, ${ }^{80 a, 80 b}$ F. Legger, ${ }^{80 a}$ C. Mariotti, ${ }^{80 a}$ S. Maselli, ${ }^{80 a}$

E. Migliore ${ }^{80 \mathrm{a}, 80 \mathrm{~b}}$ V. Monaco, ${ }^{80 \mathrm{a}, 80 \mathrm{~b}}$ E. Monteil,${ }^{80 \mathrm{a}, 80 \mathrm{~b}}$ M. Monteno,${ }^{80 \mathrm{a}}$ M. M. Obertino, ${ }^{80 \mathrm{a}, 80 \mathrm{~b}}$ G. Ortona, ${ }^{80 \mathrm{a}, 80 \mathrm{~b}}$

L. Pacher, ${ }^{80 \mathrm{a}, 80 \mathrm{~b}}$ N. Pastrone, ${ }^{80 \mathrm{a}}$ M. Pelliccioni, ${ }^{80 \mathrm{a}}$ G. L. Pinna Angioni,${ }^{80 \mathrm{a}, 80 \mathrm{~b}}$ A. Romero, ${ }^{80 \mathrm{a}, 80 \mathrm{~b}}$ M. Ruspa ${ }^{80 \mathrm{a}, 80 \mathrm{c}}$

R. Salvatico,${ }^{80 a, 80 b}$ V. Sola, ${ }^{80 a}$ A. Solano, ${ }^{80 a, 80 b}$ D. Soldi ${ }^{80 a, 80 b}$ A. Staiano, ${ }^{80 a}$ D. Trocino, ${ }^{80 a, 80 b}$ S. Belforte,${ }^{81 a}$

V. Candelise, ${ }^{81 a, 81 b}$ M. Casarsa, ${ }^{81 a}$ F. Cossutti ${ }^{81 a}$ A. Da Rold,${ }^{81 a, 81 b}$ G. Della Ricca, ${ }^{81 a, 81 b}$ F. Vazzoler, ${ }^{81 a, 81 b}$ A. Zanetti, ${ }^{81 a}$ B. Kim, ${ }^{82}$ D. H. Kim, ${ }^{82}$ G. N. Kim, ${ }^{82}$ J. Lee, ${ }^{82}$ S. W. Lee, ${ }^{82}$ C. S. Moon, ${ }^{82}$ Y. D. Oh, ${ }^{82}$ S. I. Pak, ${ }^{82}$ S. Sekmen,${ }^{82}$ D. C. Son, ${ }^{82}$ Y. C. Yang, ${ }^{82}$ H. Kim, ${ }^{83}$ D. H. Moon,${ }^{83}$ G. Oh, ${ }^{83}$ B. Francois, ${ }^{84}$ T. J. Kim,${ }^{84}$ J. Park, ${ }^{84}$ S. Cho, ${ }^{85}$ S. Choi, ${ }^{85}$ Y. Go, ${ }^{85}$ S. Ha ${ }^{85}$ B. Hong ${ }^{85}$ K. Lee, ${ }^{85}$ K. S. Lee, ${ }^{85}$ J. Lim, ${ }^{85}$ J. Park ${ }^{85}$ S. K. Park ${ }^{85}$ Y. Roh, ${ }^{85}$ J. Yoo, ${ }^{85}$ J. Goh, ${ }^{86}$ H. S. Kim, ${ }^{87}$ J. Almond ${ }^{88}$ J. H. Bhyun, ${ }^{88}$ J. Choi, ${ }^{88}$ S. Jeon, ${ }^{88}$ J. Kim, ${ }^{88}$ J. S. Kim, ${ }^{88}$ H. Lee, ${ }^{88}$ K. Lee, ${ }^{88}$ S. Lee, ${ }^{88}$ K. Nam, ${ }^{88}$ M. Oh, ${ }^{88}$ S. B. Oh, ${ }^{88}$

B. C. Radburn-Smith, ${ }^{88}$ U. K. Yang, ${ }^{88}$ H. D. Yoo, ${ }^{88}$ I. Yoon, ${ }^{88}$ D. Jeon, ${ }^{89}$ J. H. Kim, ${ }^{89}$ J. S. H. Lee, ${ }^{89}$ I. C. Park, ${ }^{89}$

I. J Watson, ${ }^{89}$ Y. Choi, ${ }^{90}$ C. Hwang, ${ }^{90}$ Y. Jeong, ${ }^{90}$ J. Lee, ${ }^{90}$ Y. Lee,${ }^{90}$ I. Yu, ${ }^{90}$ V. Veckalns, ${ }^{91, h h}$ V. Dudenas,${ }^{92}$ A. Juodagalvis, ${ }^{92}$

A. Rinkevicius, ${ }^{92}$ G. Tamulaitis, ${ }^{92}$ J. Vaitkus, ${ }^{92}$ Z. A. Ibrahim, ${ }^{93}$ F. Mohamad Idris, ${ }^{93, \text { ii }}$ W. A. T. Wan Abdullah, ${ }^{93}$

M. N. Yusli, ${ }^{93}$ Z. Zolkapli, ${ }^{93}$ J. F. Benitez, ${ }^{94}$ A. Castaneda Hernandez, ${ }^{94}$ J. A. Murillo Quijada, ${ }^{94}$ L. Valencia Palomo, ${ }^{94}$ H. Castilla-Valdez, ${ }^{95}$ E. De La Cruz-Burelo, ${ }^{95}$ I. Heredia-De La Cruz, ${ }^{95, j j}$ R. Lopez-Fernandez, ${ }^{95}$ A. Sanchez-Hernandez, ${ }^{95}$ S. Carrillo Moreno, ${ }^{96}$ C. Oropeza Barrera, ${ }^{96}$ M. Ramirez-Garcia, ${ }^{96}$ F. Vazquez Valencia, ${ }^{96}$ J. Eysermans, ${ }^{97}$ I. Pedraza, ${ }^{97}$ H. A. Salazar Ibarguen, ${ }^{97}$ C. Uribe Estrada,${ }^{97}$ A. Morelos Pineda, ${ }^{98}$ J. Mijuskovic, ${ }^{99, c}$ N. Raicevic, ${ }^{99}$ D. Krofcheck, ${ }^{100}$ S. Bheesette, ${ }^{101}$ P. H. Butler, ${ }^{101}$ A. Ahmad, ${ }^{102}$ M. Ahmad, ${ }^{102}$ Q. Hassan, ${ }^{102}$ H. R. Hoorani, ${ }^{102}$ W. A. Khan, ${ }^{102}$ M. A. Shah, ${ }^{102}$ M. Shoaib,${ }^{102}$ M. Waqas, ${ }^{102}$ V. Avati, ${ }^{103}$ L. Grzanka, ${ }^{103}$ M. Malawski, ${ }^{103}$ H. Bialkowska, ${ }^{104}$ M. Bluj, ${ }^{104}$ B. Boimska,${ }^{104}$ M. Górski ${ }^{104}$ M. Kazana, ${ }^{104}$ M. Szleper, ${ }^{104}$ P. Zalewski, ${ }^{104}$ K. Bunkowski, ${ }^{105}$ A. Byszuk, ${ }^{105, k k}$ K. Doroba, ${ }^{105}$

A. Kalinowski, ${ }^{105}$ M. Konecki, ${ }^{105}$ J. Krolikowski, ${ }^{105}$ M. Olszewski, ${ }^{105}$ M. Walczak, ${ }^{105}$ M. Araujo, ${ }^{106}$ P. Bargassa, ${ }^{106}$ D. Bastos, ${ }^{106}$ A. Di Francesco, ${ }^{106}$ P. Faccioli ${ }^{106}$ B. Galinhas, ${ }^{106}$ M. Gallinaro, ${ }^{106}$ J. Hollar, ${ }^{106}$ N. Leonardo, ${ }^{106}$ T. Niknejad, ${ }^{106}$ J. Seixas, ${ }^{106}$ K. Shchelina, ${ }^{106}$ G. Strong, ${ }^{106}$ O. Toldaiev, ${ }^{106}$ J. Varela, ${ }^{106}$ S. Afanasiev,${ }^{107}$ P. Bunin, ${ }^{107}$ M. Gavrilenko, ${ }^{107}$ I. Golutvin, ${ }^{107}$ I. Gorbunov, ${ }^{107}$ A. Kamenev, ${ }^{107}$ V. Karjavine, ${ }^{107}$ A. Lanev, ${ }^{107}$ A. Malakhov, ${ }^{107}$ V. Matveev, ${ }^{107,11, m m}$

P. Moisenz, ${ }^{107}$ V. Palichik, ${ }^{107}$ V. Perelygin, ${ }^{107}$ M. Savina, ${ }^{107}$ S. Shmatov,${ }^{107}$ S. Shulha, ${ }^{107}$ N. Skatchkov,${ }^{107}$ V. Smirnov, ${ }^{107}$ N. Voytishin, ${ }^{107}$ A. Zarubin, ${ }^{107}$ L. Chtchipounov, ${ }^{108}$ V. Golovtcov,${ }^{108}$ Y. Ivanov, ${ }^{108}$ V. Kim, ${ }^{108, n n}$ E. Kuznetsova, ${ }^{108,00}$ P. Levchenko, ${ }^{108}$ V. Murzin, ${ }^{108}$ V. Oreshkin, ${ }^{108}$ I. Smirnov, ${ }^{108}$ D. Sosnov, ${ }^{108}$ V. Sulimov, ${ }^{108}$ L. Uvarov, ${ }^{108}$ A. Vorobyev, ${ }^{108}$ Yu. Andreev, ${ }^{109}$ A. Dermenev, ${ }^{109}$ S. Gninenko, ${ }^{109}$ N. Golubev, ${ }^{109}$ A. Karneyeu, ${ }^{109}$ M. Kirsanov, ${ }^{109}$ N. Krasnikov, ${ }^{109}$

A. Pashenkov, ${ }^{109}$ D. Tlisov, ${ }^{109}$ A. Toropin, ${ }^{109}$ V. Epshteyn, ${ }^{110}$ V. Gavrilov,${ }^{110}$ N. Lychkovskaya, ${ }^{110}$ A. Nikitenko, ${ }^{110, p p}$ V. Popov ${ }^{110}$ I. Pozdnyakov, ${ }^{110}$ G. Safronov, ${ }^{110}$ A. Spiridonov ${ }^{110}$ A. Stepennov, ${ }^{110}$ M. Toms ${ }^{110}$ E. Vlasov, ${ }^{110}$ A. Zhokin, ${ }^{110}$ T. Aushev, ${ }^{111}$ O. Bychkova, ${ }^{112}$ R. Chistov, ${ }^{112, q q}$ S. Polikarpov, ${ }^{112, q q}$ E. Tarkovskii, ${ }^{112}$ E. Zhemchugov, ${ }^{112}$ V. Andreev, ${ }^{113}$ M. Azarkin, ${ }^{113}$ I. Dremin, ${ }^{113}$ M. Kirakosyan, ${ }^{113}$ A. Terkulov, ${ }^{113}$ A. Belyaev,${ }^{114}$ E. Boos, ${ }^{114}$ V. Bunichev, ${ }^{114}$ M. Dubinin, ${ }^{114, \text { rr }}$ L. Dudko, ${ }^{114}$ A. Ershov, ${ }^{114}$ A. Gribushin, ${ }^{114}$ V. Klyukhin, ${ }^{114}$ O. Kodolova, ${ }^{114}$ I. Lokhtin, ${ }^{114}$ S. Obraztsov, ${ }^{114}$ M. Perfilov, ${ }^{114}$ V. Savrin, ${ }^{114}$ A. Barnyakov, ${ }^{115, \text { ss }}$ V. Blinov, ${ }^{115, \text { ss }}$ T. Dimova, ${ }^{115, s s}$ L. Kardapoltsev, ${ }^{115, \text { ss }}$ Y. Skovpen, ${ }^{115, s s}$ I. Azhgirey, ${ }^{116}$

I. Bayshev, ${ }^{116}$ S. Bitioukov, ${ }^{116}$ V. Kachanov, ${ }^{116}$ D. Konstantinov, ${ }^{116}$ P. Mandrik, ${ }^{116}$ V. Petrov, ${ }^{116}$ R. Ryutin, ${ }^{116}$

S. Slabospitskii, ${ }^{116}$ A. Sobol, ${ }^{116}$ S. Troshin, ${ }^{116}$ N. Tyurin, ${ }^{116}$ A. Uzunian, ${ }^{116}$ A. Volkov, ${ }^{116}$ A. Babaev, ${ }^{117}$ A. Iuzhakov, ${ }^{117}$ V. Okhotnikov, ${ }^{117}$ V. Borchsh, ${ }^{118}$ V. Ivanchenko, ${ }^{118}$ E. Tcherniaev, ${ }^{118}$ P. Adzic, ${ }^{119, t t}$ P. Cirkovic, ${ }^{119}$ M. Dordevic, ${ }^{119}$ P. Milenovic, ${ }^{119}$ J. Milosevic, ${ }^{119}$ M. Stojanovic, ${ }^{119}$ M. Aguilar-Benitez, ${ }^{120}$ J. Alcaraz Maestre, ${ }^{120}$ A. Álvarez Fernández, ${ }^{120}$ I. Bachiller, ${ }^{120}$ M. Barrio Luna, ${ }^{120}$ Cristina F. Bedoya, ${ }^{120}$ J. A. Brochero Cifuentes, ${ }^{120}$ C. A. Carrillo Montoya, ${ }^{120}$ M. Cepeda, ${ }^{120}$ M. Cerrada, ${ }^{120}$ N. Colino, ${ }^{120}$ B. De La Cruz,${ }^{120}$ A. Delgado Peris, ${ }^{120}$ J. P. Fernández Ramos, ${ }^{120}$ J. Flix, ${ }^{120}$ M. C. Fouz, ${ }^{120}$ O. Gonzalez Lopez, ${ }^{120}$ S. Goy Lopez, ${ }^{120}$ J. M. Hernandez, ${ }^{120}$ M. I. Josa, ${ }^{120}$ D. Moran, ${ }^{120}$ Á. Navarro Tobar, ${ }^{120}$ A. Pérez-Calero Yzquierdo, ${ }^{120}$ J. Puerta Pelayo, ${ }^{120}$ I. Redondo, ${ }^{120}$ L. Romero, ${ }^{120}$ S. Sánchez Navas, ${ }^{120}$ M. S. Soares,${ }^{120}$ A. Triossi, ${ }^{120}$ C. Willmott, ${ }^{120}$ C. Albajar, ${ }^{121}$ J. F. de Trocóniz, ${ }^{121}$ R. Reyes-Almanza, ${ }^{121}$ B. Alvarez Gonzalez, ${ }^{122}$ J. Cuevas, ${ }^{122}$

C. Erice, ${ }^{122}$ J. Fernandez Menendez, ${ }^{122}$ S. Folgueras, ${ }^{122}$ I. Gonzalez Caballero, ${ }^{122}$ J. R. González Fernández, ${ }^{122}$

E. Palencia Cortezon, ${ }^{122}$ V. Rodríguez Bouza, ${ }^{122}$ S. Sanchez Cruz, ${ }^{122}$ I. J. Cabrillo, ${ }^{123}$ A. Calderon, ${ }^{123}$ B. Chazin Quero, ${ }^{123}$ J. Duarte Campderros, ${ }^{123}$ M. Fernandez, ${ }^{123}$ P. J. Fernández Manteca, ${ }^{123}$ A. García Alonso, ${ }^{123}$ G. Gomez, ${ }^{123}$ 
C. Martinez Rivero, ${ }^{123}$ P. Martinez Ruiz del Arbol, ${ }^{123}$ F. Matorras, ${ }^{123}$ J. Piedra Gomez, ${ }^{123}$ C. Prieels, ${ }^{123}$ T. Rodrigo, ${ }^{123}$ A. Ruiz-Jimeno, ${ }^{123}$ L. Russo, ${ }^{123, \text { uu }}$ L. Scodellaro, ${ }^{123}$ I. Vila, ${ }^{123}$ J. M. Vizan Garcia, ${ }^{123}$ K. Malagalage, ${ }^{124}$ W. G. D. Dharmaratna, ${ }^{125}$ N. Wickramage, ${ }^{125}$ D. Abbaneo,${ }^{126}$ B. Akgun, ${ }^{126}$ E. Auffray, ${ }^{126}$ G. Auzinger, ${ }^{126}$ J. Baechler, ${ }^{126}$ P. Baillon, ${ }^{126}$ A. H. Ball, ${ }^{126}$ D. Barney, ${ }^{126}$ J. Bendavid, ${ }^{126}$ M. Bianco, ${ }^{126}$ A. Bocci, ${ }^{126}$ P. Bortignon, ${ }^{126}$ E. Bossini, ${ }^{126}$ C. Botta, ${ }^{126}$ E. Brondolin, ${ }^{126}$ T. Camporesi, ${ }^{126}$ A. Caratelli, ${ }^{126}$ G. Cerminara, ${ }^{126}$ E. Chapon, ${ }^{126}$ G. Cucciati, ${ }^{126}$ D. d'Enterria, ${ }^{126}$ A. Dabrowski, ${ }^{126}$ N. Daci ${ }^{126}$ V. Daponte, ${ }^{126}$ A. David, ${ }^{126}$ O. Davignon, ${ }^{126}$ A. De Roeck, ${ }^{126}$ M. Deile, ${ }^{126}$ M. Dobson, ${ }^{126}$ M. Dünser, ${ }^{126}$ N. Dupont, ${ }^{126}$ A. Elliott-Peisert, ${ }^{126}$ N. Emriskova, ${ }^{126}$ F. Fallavollita, ${ }^{126, v v}$ D. Fasanella, ${ }^{126}$ S. Fiorendi, ${ }^{126}$ G. Franzoni, ${ }^{126}$ J. Fulcher, ${ }^{126}$ W. Funk, ${ }^{126}$ S. Giani, ${ }^{126}$ D. Gigi, ${ }^{126}$ A. Gilbert, ${ }^{126}$ K. Gill, ${ }^{126}$ F. Glege, ${ }^{126}$ L. Gouskos, ${ }^{126}$ M. Gruchala, ${ }^{126}$ M. Guilbaud, ${ }^{126}$ D. Gulhan, ${ }^{126}$ J. Hegeman, ${ }^{126}$ C. Heidegger, ${ }^{126}$ Y. Iiyama, ${ }^{126}$ V. Innocente, ${ }^{126}$ T. James, ${ }^{126}$ P. Janot, ${ }^{126}$ O. Karacheban, ${ }^{126, t}$ J. Kaspar, ${ }^{126}$ J. Kieseler, ${ }^{126}$ M. Krammer, ${ }^{126, b}$ N. Kratochwil, ${ }^{126}$ C. Lange, ${ }^{126}$ P. Lecoq, ${ }^{126}$ C. Lourenço, ${ }^{126}$ L. Malgeri, ${ }^{126}$ M. Mannelli, ${ }^{126}$ A. Massironi, ${ }^{126}$ F. Meijers, ${ }^{126}$ J. A. Merlin, ${ }^{126}$ S. Mersi, ${ }^{126}$ E. Meschi, ${ }^{126}$ F. Moortgat, ${ }^{126}$ M. Mulders, ${ }^{126}$ J. Ngadiuba, ${ }^{126}$ J. Niedziela ${ }^{126}$ S. Nourbakhsh, ${ }^{126}$ S. Orfanelli, ${ }^{126}$ L. Orsini ${ }^{126}$ F. Pantaleo, ${ }^{126, \mathrm{q}}$ L. Pape,${ }^{126}$ E. Perez, ${ }^{126}$ M. Peruzzi, ${ }^{126}$ A. Petrilli, ${ }^{126}$ G. Petrucciani, ${ }^{126}$ A. Pfeiffer, ${ }^{126}$ M. Pierini, ${ }^{126}$ F. M. Pitters, ${ }^{126}$ D. Rabady, ${ }^{126}$ A. Racz, ${ }^{126}$ M. Rieger, ${ }^{126}$ M. Rovere, ${ }^{126}$ H. Sakulin, ${ }^{126}$ J. Salfeld-Nebgen, ${ }^{126}$ C. Schäfer, ${ }^{126}$ C. Schwick, ${ }^{126}$ M. Selvaggi, ${ }^{126}$ A. Sharma, ${ }^{126}$ P. Silva, ${ }^{126}$ W. Snoeys, ${ }^{126}$ P. Sphicas, ${ }^{126, w w}$ J. Steggemann, ${ }^{126}$ S. Summers, ${ }^{126}$ V. R. Tavolaro, ${ }^{126}$ D. Treille, ${ }^{126}$ A. Tsirou, ${ }^{126}$ G. P. Van Onsem, ${ }^{126}$ A. Vartak, ${ }^{126}$ M. Verzetti, ${ }^{126}$ W. D. Zeuner, ${ }^{126}$ L. Caminada, ${ }^{127, x x}$ K. Deiters, ${ }^{127}$ W. Erdmann, ${ }^{127}$ R. Horisberger ${ }^{127}$ Q. Ingram, ${ }^{127}$ H. C. Kaestli, ${ }^{127}$ D. Kotlinski, ${ }^{127}$ U. Langenegger, ${ }^{127}$ T. Rohe, ${ }^{127}$ S. A. Wiederkehr, ${ }^{127}$ M. Backhaus, ${ }^{128}$ P. Berger, ${ }^{128}$ N. Chernyavskaya, ${ }^{128}$ G. Dissertori, ${ }^{128}$ M. Dittmar, ${ }^{128}$ M. Donegà, ${ }^{128}$ C. Dorfer, ${ }^{128}$ T. A. Gómez Espinosa, ${ }^{128}$ C. Grab,${ }^{128}$ D. Hits, ${ }^{128}$ W. Lustermann, ${ }^{128}$ R. A. Manzoni, ${ }^{128}$ M. T. Meinhard, ${ }^{128}$ F. Micheli, ${ }^{128}$ P. Musella, ${ }^{128}$ F. Nessi-Tedaldi, ${ }^{128}$ F. Pauss, ${ }^{128}$ G. Perrin, ${ }^{128}$ L. Perrozzi, ${ }^{128}$ S. Pigazzini, ${ }^{128}$ M. G. Ratti, ${ }^{128}$ M. Reichmann, ${ }^{128}$ C. Reissel, ${ }^{128}$ T. Reitenspiess,${ }^{128}$ B. Ristic, ${ }^{128}$ D. Ruini, ${ }^{128}$ D. A. Sanz Becerra, ${ }^{128}$ M. Schönenberger, ${ }^{128}$ L. Shchutska, ${ }^{128}$ M. L. Vesterbacka Olsson, ${ }^{128}$ R. Wallny, ${ }^{128}$ D. H. Zhu, ${ }^{128}$ T. K. Aarrestad, ${ }^{129}$ C. Amsler, ${ }^{129, y y}$ D. Brzhechko, ${ }^{129}$ M. F. Canelli, ${ }^{129}$ A. De Cosa, ${ }^{129}$ R. Del Burgo, ${ }^{129}$ B. Kilminster, ${ }^{129}$ S. Leontsinis, ${ }^{129}$ V. M. Mikuni, ${ }^{129}$ I. Neutelings, ${ }^{129}$ G. Rauco, ${ }^{129}$ P. Robmann, ${ }^{129}$ K. Schweiger, ${ }^{129}$ C. Seitz ${ }^{129}$ Y. Takahashi, ${ }^{129}$ S. Wertz ${ }^{129}$ A. Zucchetta, ${ }^{129}$ T. H. Doan, ${ }^{130}$ C. M. Kuo, ${ }^{130}$ W. Lin,${ }^{130}$ A. Roy, ${ }^{130}$ S. S. Yu, ${ }^{130}$ P. Chang, ${ }^{131}$ Y. Chao, ${ }^{131}$ K. F. Chen, ${ }^{131}$ P. H. Chen, ${ }^{131}$ W.-S. Hou, ${ }^{131}$ Y. y. Li, ${ }^{131}$ R.-S. Lu, ${ }^{131}$ E. Paganis, ${ }^{131}$ A. Psallidas, ${ }^{131}$ A. Steen, ${ }^{131}$ B. Asavapibhop, ${ }^{132}$ C. Asawatangtrakuldee, ${ }^{132}$ N. Srimanobhas, ${ }^{132}$ N. Suwonjandee, ${ }^{132}$ A. Bat,${ }^{133}$ F. Boran, ${ }^{133}$ A. Celik, ${ }^{133, \text { zz }}$ S. Cerci, ${ }^{133, \text { aaa }}$ S. Damarseckin, ${ }^{133, \text { bbb }}$ Z. S. Demiroglu, ${ }^{133}$ F. Dolek, ${ }^{133}$ C. Dozen, ${ }^{133, c c c}$ I. Dumanoglu, ${ }^{133}$ G. Gokbulut, ${ }^{133}$ Emine Gurpinar Guler, ${ }^{133, \text { ddd }}$ Y. Guler, ${ }^{133}$ I. Hos, ${ }^{133, \text { eee }}$ C. Isik, ${ }^{133}$ E. E. Kangal, ${ }^{133, f f f}$ O. Kara, ${ }^{133}$ A. Kayis Topaksu, ${ }^{133}$ U. Kiminsu, ${ }^{133}$ G. Onengut, ${ }^{133}$ K. Ozdemir, ${ }^{133, g g g}$ S. Ozturk, ${ }^{133, \text { hhh }}$ A. E. Simsek, ${ }^{133}$ D. Sunar Cerci, ${ }^{133, \text { aaa }}$ U. G. Tok, ${ }^{133}$ S. Turkcapar, ${ }^{133}$ I. S. Zorbakir, ${ }^{133}$ C. Zorbilmez, ${ }^{133}$ B. Isildak, ${ }^{134, \text { iii }}$ G. Karapinar, ${ }^{134, j j j}$ M. Yalvac, ${ }^{134}$ I. O. Atakisi, ${ }^{135}$ E. Gülmez, ${ }^{135}$ M. Kaya ${ }^{135, k k k}$ O. Kaya,${ }^{135,111}$ Ö. Özçelik, ${ }^{135}$ S. Tekten, ${ }^{135}$ E. A. Yetkin, ${ }^{135, \mathrm{mmm}}$ A. Cakir, ${ }^{136}$ K. Cankocak, ${ }^{136}$ Y. Komurcu, ${ }^{136}$ S. Sen, ${ }^{136, n n n}$ B. Kaynak, ${ }^{137}$ S. Ozkorucuklu, ${ }^{137}$ B. Grynyov, ${ }^{138}$ L. Levchuk, ${ }^{139}$ E. Bhal, ${ }^{140}$ S. Bologna, ${ }^{140}$ J. J. Brooke, ${ }^{140}$ D. Burns, ${ }^{140,000}$ E. Clement, ${ }^{140}$ D. Cussans, ${ }^{140}$ H. Flacher, ${ }^{140}$ J. Goldstein, ${ }^{140}$ G. P. Heath ${ }^{140}$ H. F. Heath, ${ }^{140}$ L. Kreczko, ${ }^{140}$ B. Krikler, ${ }^{140}$ S. Paramesvaran, ${ }^{140}$ B. Penning,,${ }^{140}$ T. Sakuma, ${ }^{140}$ S. Seif El Nasr-Storey, ${ }^{140}$ V. J. Smith, ${ }^{140}$ J. Taylor, ${ }^{140}$ A. Titterton, ${ }^{140}$ K. W. Bell ${ }^{141}$ A. Belyaev, ${ }^{141, p p p}$ C. Brew, ${ }^{141}$ R. M. Brown, ${ }^{141}$ D. J. A. Cockerill, ${ }^{141}$ J. A. Coughlan, ${ }^{141}$ K. Harder ${ }^{141}$ S. Harper, ${ }^{141}$ J. Linacre, ${ }^{141}$ K. Manolopoulos ${ }^{141}$ D. M. Newbold, ${ }^{141}$ E. Olaiya, ${ }^{141}$ D. Petyt, ${ }^{141}$ T. Reis, ${ }^{141}$ T. Schuh,${ }^{141}$ C. H. Shepherd-Themistocleous, ${ }^{141}$ A. Thea, ${ }^{141}$

I. R. Tomalin, ${ }^{141}$ T. Williams, ${ }^{141}$ W. J. Womersley, ${ }^{141}$ R. Bainbridge, ${ }^{142}$ P. Bloch, ${ }^{142}$ J. Borg, ${ }^{142}$ S. Breeze,${ }^{142}$ O. Buchmuller, ${ }^{142}$ A. Bundock, ${ }^{142}$ Gurpreet Singh CHAHAL, ${ }^{142, q q q}$ D. Colling, ${ }^{142}$ P. Dauncey, ${ }^{142}$ G. Davies,${ }^{142}$ M. Della Negra, ${ }^{142}$ R. Di Maria, ${ }^{142}$ P. Everaerts, ${ }^{142}$ G. Hall, ${ }^{142}$ G. Iles, ${ }^{142}$ M. Komm, ${ }^{142}$ C. Laner, ${ }^{142}$ L. Lyons, ${ }^{142}$ A.-M. Magnan, ${ }^{142}$ S. Malik, ${ }^{142}$ A. Martelli, ${ }^{142}$ V. Milosevic, ${ }^{142}$ A. Morton, ${ }^{142}$ J. Nash,${ }^{142, \text { rrr }}$ V. Palladino, ${ }^{142}$ M. Pesaresi, ${ }^{142}$ D. M. Raymond, ${ }^{142}$ A. Richards, ${ }^{142}$ A. Rose,${ }^{142}$ E. Scott, ${ }^{142}$ C. Seez, ${ }^{142}$ A. Shtipliyski, ${ }^{142}$ M. Stoye, ${ }^{142}$ T. Strebler,${ }^{142}$

A. Tapper, ${ }^{142}$ K. Uchida, ${ }^{142}$ T. Virdee,${ }^{142, q}$ N. Wardle, ${ }^{142}$ D. Winterbottom, ${ }^{142}$ J. Wright, ${ }^{142}$ A. G. Zecchinelli, ${ }^{142}$ S. C. Zenz ${ }^{142}$ J. E. Cole, ${ }^{143}$ P. R. Hobson, ${ }^{143}$ A. Khan, ${ }^{143}$ P. Kyberd, ${ }^{143}$ C. K. Mackay, ${ }^{143}$ I. D. Reid, ${ }^{143}$ L. Teodorescu, ${ }^{143}$ S. Zahid, ${ }^{143}$ K. Call, ${ }^{144}$ B. Caraway, ${ }^{144}$ J. Dittmann, ${ }^{144}$ K. Hatakeyama, ${ }^{144}$ C. Madrid, ${ }^{144}$ B. McMaster, ${ }^{144}$ N. Pastika, ${ }^{144}$ C. Smith, ${ }^{144}$ R. Bartek, ${ }^{145}$ A. Dominguez, ${ }^{145}$ R. Uniyal,${ }^{145}$ A. M. Vargas Hernandez, ${ }^{145}$ A. Buccilli, ${ }^{146}$ S. I. Cooper, ${ }^{146}$ C. Henderson, ${ }^{146}$ P. Rumerio, ${ }^{146}$ C. West, ${ }^{146}$ A. Albert,${ }^{147}$ D. Arcaro, ${ }^{147}$ Z. Demiragli, ${ }^{147}$ D. Gastler,${ }^{147}$ C. Richardson, ${ }^{147}$ J. Rohlf, ${ }^{147}$ D. Sperka, ${ }^{147}$ I. Suarez, ${ }^{147}$ L. Sulak, ${ }^{147}$ D. Zou, ${ }^{147}$ G. Benelli, ${ }^{148}$ B. Burkle, ${ }^{148}$ X. Coubez, ${ }^{148, \mathrm{r}}$ D. Cutts, ${ }^{148}$ 
Y. t. Duh, ${ }^{148}$ M. Hadley, ${ }^{148}$ U. Heintz, ${ }^{148}$ J. M. Hogan, ${ }^{148, \text { sss }}$ K. H. M. Kwok, ${ }^{148}$ E. Laird, ${ }^{148}$ G. Landsberg, ${ }^{148}$ K. T. Lau, ${ }^{148}$ J. Lee, ${ }^{148}$ Z. Mao,${ }^{148}$ M. Narain, ${ }^{148}$ S. Sagir, ${ }^{148, \text { tt }}$ R. Syarif, ${ }^{148}$ E. Usai,${ }^{148}$ D. Yu,${ }^{148}$ W. Zhang, ${ }^{148}$ R. Band,${ }^{149}$ C. Brainerd, ${ }^{149}$ R. Breedon, ${ }^{149}$ M. Calderon De La Barca Sanchez, ${ }^{149}$ M. Chertok, ${ }^{149}$ J. Conway, ${ }^{149}$ R. Conway, ${ }^{149}$ P. T. Cox,${ }^{149}$ R. Erbacher ${ }^{149}$ C. Flores, ${ }^{149}$ G. Funk,${ }^{149}$ F. Jensen, ${ }^{149}$ W. Ko, ${ }^{149}$ O. Kukral, ${ }^{149}$ R. Lander, ${ }^{149}$ M. Mulhearn, ${ }^{149}$ D. Pellett, ${ }^{149}$ J. Pilot, ${ }^{149}$ M. Shi, ${ }^{149}$ D. Taylor, ${ }^{149}$ K. Tos, ${ }^{149}$ M. Tripathi, ${ }^{149}$ Z. Wang,${ }^{149}$ F. Zhang, ${ }^{149}$ M. Bachtis, ${ }^{150}$ C. Bravo, ${ }^{150}$ R. Cousins, ${ }^{150}$ A. Dasgupta, ${ }^{150}$ A. Florent, ${ }^{150}$ J. Hauser, ${ }^{150}$ M. Ignatenko, ${ }^{150}$ N. Mccoll, ${ }^{150}$ W. A. Nash, ${ }^{150}$ S. Regnard, ${ }^{150}$ D. Saltzberg, ${ }^{150}$ C. Schnaible, ${ }^{150}$ B. Stone, ${ }^{150}$ V. Valuev,${ }^{150}$ K. Burt, ${ }^{151}$ Y. Chen, ${ }^{151}$ R. Clare, ${ }^{151}$ J. W. Gary, ${ }^{151}$ S. M. A. Ghiasi Shirazi, ${ }^{151}$ G. Hanson, ${ }^{151}$ G. Karapostoli, ${ }^{151}$ E. Kennedy, ${ }^{151}$ O. R. Long, ${ }^{151}$ M. Olmedo Negrete, ${ }^{151}$ M. I. Paneva, ${ }^{151}$ W. Si, ${ }^{151}$ L. Wang, ${ }^{151}$ S. Wimpenny, ${ }^{151}$ B. R. Yates, ${ }^{151}$ Y. Zhang, ${ }^{151}$ J. G. Branson, ${ }^{152}$ P. Chang, ${ }^{152}$ S. Cittolin, ${ }^{152}$ S. Cooperstein, ${ }^{152}$ N. Deelen, ${ }^{152}$ M. Derdzinski, ${ }^{152}$ R. Gerosa,${ }^{152}$ D. Gilbert, ${ }^{152}$ B. Hashemi, ${ }^{152}$ D. Klein, ${ }^{152}$ V. Krutelyov, ${ }^{152}$ J. Letts, ${ }^{152}$ M. Masciovecchio, ${ }^{152}$ S. May, ${ }^{152}$ S. Padhi,${ }^{152}$ M. Pieri, ${ }^{152}$ V. Sharma,${ }^{152}$ M. Tadel, ${ }^{152}$ F. Würthwein, ${ }^{152}$ A. Yagil,${ }^{152}$ G. Zevi Della Porta, ${ }^{152}$ N. Amin, ${ }^{153}$ R. Bhandari, ${ }^{153}$ C. Campagnari,${ }^{153}$ M. Citron, ${ }^{153}$ V. Dutta, ${ }^{153}$ M. Franco Sevilla, ${ }^{153}$ J. Incandela, ${ }^{153}$ B. Marsh ${ }^{153}$ H. Mei, ${ }^{153}$ A. Ovcharova, ${ }^{153}$ H. Qu, ${ }^{153}$ J. Richman, ${ }^{153}$ U. Sarica, ${ }^{153}$ D. Stuart, ${ }^{153}$ S. Wang, ${ }^{153}$ D. Anderson, ${ }^{154}$ A. Bornheim, ${ }^{154}$ O. Cerri, ${ }^{154}$ I. Dutta, ${ }^{154}$ J. M. Lawhorn, ${ }^{154}$ N. Lu, ${ }^{154}$ J. Mao, ${ }^{154}$ H. B. Newman, ${ }^{154}$ T. Q. Nguyen, ${ }^{154}$ J. Pata, ${ }^{154}$ M. Spiropulu, ${ }^{154}$ J. R. Vlimant, ${ }^{154}$ S. Xie, ${ }^{154}$ Z. Zhang, ${ }^{154}$ R. Y. Zhu, ${ }^{154}$ M. B. Andrews, ${ }^{155}$ T. Ferguson, ${ }^{155}$ T. Mudholkar, ${ }^{155}$ M. Paulini, ${ }^{155}$ M. Sun ${ }^{155}$ I. Vorobiev, ${ }^{155}$ M. Weinberg, ${ }^{155}$ J. P. Cumalat, ${ }^{156}$ W. T. Ford, ${ }^{156}$ E. MacDonald, ${ }^{156}$ T. Mulholland, ${ }^{156}$ R. Patel, ${ }^{156}$ A. Perloff, ${ }^{156}$ K. Stenson, ${ }^{156}$ K. A. Ulmer, ${ }^{156}$ S. R. Wagner, ${ }^{156}$ J. Alexander, ${ }^{157}$ Y. Cheng, ${ }^{157}$ J. Chu, ${ }^{157}$ A. Datta, ${ }^{157}$ A. Frankenthal, ${ }^{157}$ K. Mcdermott, ${ }^{157}$ J. R. Patterson, ${ }^{157}$ D. Quach, ${ }^{157}$ A. Ryd ${ }^{157}$ S. M. Tan, ${ }^{157}$ Z. Tao, ${ }^{157}$ J. Thom, ${ }^{157}$ P. Wittich, ${ }^{157}$ M. Zientek, ${ }^{157}$ S. Abdullin, ${ }^{158}$ M. Albrow, ${ }^{158}$ M. Alyari, ${ }^{158}$ G. Apollinari, ${ }^{158}$ A. Apresyan, ${ }^{158}$ A. Apyan, ${ }^{158}$ S. Banerjee, ${ }^{158}$ L. A. T. Bauerdick, ${ }^{158}$ A. Beretvas, ${ }^{158}$ D. Berry, ${ }^{158}$ J. Berryhill, ${ }^{158}$ P. C. Bhat, ${ }^{158}$ K. Burkett, ${ }^{158}$ J. N. Butler, ${ }^{158}$ A. Canepa, ${ }^{158}$ G. B. Cerati, ${ }^{158}$ H. W. K. Cheung, ${ }^{158}$ F. Chlebana, ${ }^{158}$ M. Cremonesi, ${ }^{158}$ J. Duarte, ${ }^{158}$ V. D. Elvira, ${ }^{158}$ J. Freeman, ${ }^{158}$ Z. Gecse, ${ }^{158}$ E. Gottschalk, ${ }^{158}$ L. Gray, ${ }^{158}$ D. Green, ${ }^{158}$ S. Grünendahl, ${ }^{158}$ O. Gutsche, ${ }^{158}$ Allison Reinsvold Hall, ${ }^{158}$ J. Hanlon, ${ }^{158}$ R. M. Harris, ${ }^{158}$ S. Hasegawa, ${ }^{158}$ R. Heller ${ }^{158}$ J. Hirschauer, ${ }^{158}$ B. Jayatilaka, ${ }^{158}$ S. Jindariani, ${ }^{158}$ M. Johnson, ${ }^{158}$ U. Joshi, ${ }^{158}$ T. Klijnsma,${ }^{158}$ B. Klima, ${ }^{158}$ M. J. Kortelainen, ${ }^{158}$ B. Kreis, ${ }^{158}$ S. Lammel, ${ }^{158}$ J. Lewis, ${ }^{158}$ D. Lincoln, ${ }^{158}$ R. Lipton, ${ }^{158}$ M. Liu, ${ }^{158}$ T. Liu, ${ }^{158}$ J. Lykken, ${ }^{158}$ K. Maeshima, ${ }^{158}$ J. M. Marraffino, ${ }^{158}$ D. Mason, ${ }^{158}$ P. McBride, ${ }^{158}$ P. Merkel, ${ }^{158}$ S. Mrenna, ${ }^{158}$ S. Nahn, ${ }^{158}$ V. O'Dell, ${ }^{158}$ V. Papadimitriou, ${ }^{158}$ K. Pedro, ${ }^{158}$ C. Pena,${ }^{158}$ G. Rakness, ${ }^{158}$ F. Ravera ${ }^{158}$ L. Ristori, ${ }^{158}$ B. Schneider,${ }^{158}$

E. Sexton-Kennedy, ${ }^{158}$ N. Smith, ${ }^{158}$ A. Soha, ${ }^{158}$ W. J. Spalding, ${ }^{158}$ L. Spiegel, ${ }^{158}$ S. Stoynev, ${ }^{158}$ J. Strait, ${ }^{158}$ N. Strobbe, ${ }^{158}$ L. Taylor, ${ }^{158}$ S. Tkaczyk, ${ }^{158}$ N. V. Tran, ${ }^{158}$ L. Uplegger, ${ }^{158}$ E. W. Vaandering, ${ }^{158}$ C. Vernieri, ${ }^{158}$ R. Vidal,${ }^{158}$ M. Wang, ${ }^{158}$ H. A. Weber, ${ }^{158}$ D. Acosta, ${ }^{159}$ P. Avery, ${ }^{159}$ D. Bourilkov, ${ }^{159}$ A. Brinkerhoff, ${ }^{159}$ L. Cadamuro, ${ }^{159}$ A. Carnes, ${ }^{159}$ V. Cherepanov, ${ }^{159}$ F. Errico, ${ }^{159}$ R. D. Field, ${ }^{159}$ S. V. Gleyzer, ${ }^{159}$ B. M. Joshi, ${ }^{159}$ M. Kim, ${ }^{159}$ J. Konigsberg, ${ }^{159}$ A. Korytov, ${ }^{159}$ K. H. Lo, ${ }^{159}$ P. Ma, ${ }^{159}$ K. Matchev, ${ }^{159}$ N. Menendez, ${ }^{159}$ G. Mitselmakher, ${ }^{159}$ D. Rosenzweig, ${ }^{159}$ K. Shi, ${ }^{159}$ J. Wang, ${ }^{159}$ S. Wang, ${ }^{159}$ X. Zuo, ${ }^{159}$ Y. R. Joshi, ${ }^{160}$ T. Adams, ${ }^{161}$ A. Askew, ${ }^{161}$ S. Hagopian, ${ }^{161}$ V. Hagopian, ${ }^{161}$ K. F. Johnson, ${ }^{161}$

R. Khurana, ${ }^{161}$ T. Kolberg, ${ }^{161}$ G. Martinez, ${ }^{161}$ T. Perry, ${ }^{161}$ H. Prosper, ${ }^{161}$ C. Schiber ${ }^{161}$ R. Yohay, ${ }^{161}$ J. Zhang, ${ }^{161}$ M. M. Baarmand, ${ }^{162}$ M. Hohlmann ${ }^{162}$ D. Noonan, ${ }^{162}$ M. Rahmani, ${ }^{162}$ M. Saunders, ${ }^{162}$ F. Yumiceva, ${ }^{162}$ M. R. Adams, ${ }^{163}$ L. Apanasevich, ${ }^{163}$ R. R. Betts, ${ }^{163}$ R. Cavanaugh, ${ }^{163}$ X. Chen, ${ }^{163}$ S. Dittmer, ${ }^{163}$ O. Evdokimov, ${ }^{163}$ C. E. Gerber, ${ }^{163}$ D. A. Hangal, ${ }^{163}$ D. J. Hofman, ${ }^{163}$ K. Jung, ${ }^{163}$ C. Mills, ${ }^{163}$ T. Roy, ${ }^{163}$ M. B. Tonjes, ${ }^{163}$ N. Varelas, ${ }^{163}$ J. Viinikainen, ${ }^{163}$

H. Wang, ${ }^{163}$ X. Wang, ${ }^{163}$ Z. Wu, ${ }^{163}$ M. Alhusseini, ${ }^{164}$ B. Bilki, ${ }^{164, d d d}$ W. Clarida, ${ }^{164}$ K. Dilsiz, ${ }^{164, \text { uuu }}$ S. Durgut, ${ }^{164}$ R. P. Gandrajula, ${ }^{164}$ M. Haytmyradov, ${ }^{164}$ V. Khristenko, ${ }^{164}$ O. K. Köseyan, ${ }^{164}$ J.-P. Merlo, ${ }^{164}$ A. Mestvirishvili, ${ }^{164, v v v}$ A. Moeller, ${ }^{164}$ J. Nachtman, ${ }^{164}$ H. Ogul, ${ }^{164, w w w}$ Y. Onel, ${ }^{164}$ F. Ozok, ${ }^{164, x x x}$ A. Penzo,${ }^{164}$ C. Snyder, ${ }^{164}$ E. Tiras, ${ }^{164}$ J. Wetzel, ${ }^{164}$ B. Blumenfeld, ${ }^{165}$ A. Cocoros, ${ }^{165}$ N. Eminizer, ${ }^{165}$ A. V. Gritsan, ${ }^{165}$ W. T. Hung, ${ }^{165}$ S. Kyriacou, ${ }^{165}$ P. Maksimovic, ${ }^{165}$ J. Roskes, ${ }^{165}$ M. Swartz, ${ }^{165}$ C. Baldenegro Barrera, ${ }^{166}$ P. Baringer, ${ }^{166}$ A. Bean, ${ }^{166}$ S. Boren, ${ }^{166}$ J. Bowen, ${ }^{166}$ A. Bylinkin, ${ }^{166}$ T. Isidori, ${ }^{166}$ S. Khalil, ${ }^{166}$ J. King, ${ }^{166}$ G. Krintiras, ${ }^{166}$ A. Kropivnitskaya,${ }^{166}$ C. Lindsey, ${ }^{166}$ D. Majumder, ${ }^{166}$ W. Mcbrayer, ${ }^{166}$ N. Minafra, ${ }^{166}$ M. Murray, ${ }^{166}$ C. Rogan, ${ }^{166}$ C. Royon, ${ }^{166}$ S. Sanders, ${ }^{166}$ E. Schmitz ${ }^{166}$ J. D. Tapia Takaki, ${ }^{166}$ Q. Wang, ${ }^{166}$ J. Williams, ${ }^{166}$ G. Wilson, ${ }^{166}$ S. Duric ${ }^{167}$ A. Ivanov, ${ }^{167}$ K. Kaadze, ${ }^{167}$ D. Kim, ${ }^{167}$ Y. Maravin, ${ }^{167}$ D. R. Mendis, ${ }^{167}$ T. Mitchell, ${ }^{167}$ A. Modak, ${ }^{167}$ A. Mohammadi, ${ }^{167}$ F. Rebassoo, ${ }^{168}$ D. Wright, ${ }^{168}$ A. Baden, ${ }^{169}$ O. Baron, ${ }^{169}$ A. Belloni, ${ }^{169}$ S. C. Eno, ${ }^{169}$ Y. Feng, ${ }^{169}$ N. J. Hadley, ${ }^{169}$ S. Jabeen, ${ }^{169}$ G. Y. Jeng, ${ }^{169}$ R. G. Kellogg, ${ }^{169}$ J. Kunkle, ${ }^{169}$ A. C. Mignerey, ${ }^{169}$ S. Nabili, ${ }^{169}$ F. Ricci-Tam, ${ }^{169}$ M. Seidel, ${ }^{169}$ Y. H. Shin, ${ }^{169}$ A. Skuja, ${ }^{169}$ S. C. Tonwar, ${ }^{169}$ K. Wong, ${ }^{169}$ D. Abercrombie, ${ }^{170}$ B. Allen, ${ }^{170}$ A. Baty, ${ }^{170}$ R. Bi, ${ }^{170}$ S. Brandt, ${ }^{170}$ W. Busza, ${ }^{170}$ I. A. Cali, ${ }^{170}$ M. D'Alfonso, ${ }^{170}$ G. Gomez Ceballos, ${ }^{170}$ 
M. Goncharov,${ }^{170}$ P. Harris, ${ }^{170}$ D. Hsu, ${ }^{170}$ M. Hu ${ }^{170}$ M. Klute, ${ }^{170}$ D. Kovalskyi,${ }^{170}$ Y.-J. Lee, ${ }^{170}$ P. D. Luckey, ${ }^{170}$ B. Maier, ${ }^{170}$ A. C. Marini, ${ }^{170}$ C. Mcginn, ${ }^{170}$ C. Mironov, ${ }^{170}$ S. Narayanan, ${ }^{170}$ X. Niu ${ }^{170}$ C. Paus,${ }^{170}$ D. Rankin,${ }^{170}$ C. Roland,${ }^{170}$ G. Roland, ${ }^{170}$ Z. Shi, ${ }^{170}$ G. S. F. Stephans, ${ }^{170}$ K. Sumorok, ${ }^{170}$ K. Tatar, ${ }^{170}$ D. Velicanu, ${ }^{170}$ J. Wang, ${ }^{170}$ T. W. Wang, ${ }^{170}$ B. Wyslouch, ${ }^{170}$ R. M. Chatterjee, ${ }^{171}$ A. Evans, ${ }^{171}$ S. Guts, ${ }^{171, a}$ P. Hansen, ${ }^{171}$ J. Hiltbrand, ${ }^{171}$ Sh. Jain, ${ }^{171}$ Y. Kubota, ${ }^{171}$ Z. Lesko, ${ }^{171}$ J. Mans, ${ }^{171}$ M. Revering, ${ }^{171}$ R. Rusack, ${ }^{171}$ R. Saradhy, ${ }^{171}$ N. Schroeder, ${ }^{171}$ M. A. Wadud, ${ }^{171}$ J. G. Acosta, ${ }^{172}$ S. Oliveros, ${ }^{172}$ K. Bloom, ${ }^{173}$ S. Chauhan, ${ }^{173}$ D. R. Claes, ${ }^{173}$ C. Fangmeier, ${ }^{173}$ L. Finco, ${ }^{173}$ F. Golf, ${ }^{173}$ R. Kamalieddin, ${ }^{173}$ I. Kravchenko, ${ }^{173}$ J. E. Siado, ${ }^{173}$ G. R. Snow, ${ }^{173, a}$ B. Stieger, ${ }^{173}$ W. Tabb ${ }^{173}$ G. Agarwal,${ }^{174}$ C. Harrington, ${ }^{174}$ I. Iashvili, ${ }^{174}$ A. Kharchilava, ${ }^{174}$ C. McLean, ${ }^{174}$ D. Nguyen, ${ }^{174}$ A. Parker, ${ }^{174}$ J. Pekkanen, ${ }^{174}$ S. Rappoccio, ${ }^{174}$ B. Roozbahani, ${ }^{174}$ G. Alverson, ${ }^{175}$ E. Barberis, ${ }^{175}$ C. Freer, ${ }^{175}$ Y. Haddad, ${ }^{175}$ A. Hortiangtham, ${ }^{175}$ G. Madigan, ${ }^{175}$ B. Marzocchi, ${ }^{175}$ D. M. Morse, ${ }^{175}$ T. Orimoto, ${ }^{175}$ L. Skinnari, ${ }^{175}$ A. Tishelman-Charny, ${ }^{175}$ T. Wamorkar, ${ }^{175}$ B. Wang, ${ }^{175}$ A. Wisecarver, ${ }^{175}$ D. Wood, ${ }^{175}$ S. Bhattacharya, ${ }^{176}$ J. Bueghly, ${ }^{176}$ T. Gunter, ${ }^{176}$ K. A. Hahn, ${ }^{176}$ N. Odell, ${ }^{176}$ M. H. Schmitt, ${ }^{176}$ K. Sung,${ }^{176}$ M. Trovato, ${ }^{176}$ M. Velasco, ${ }^{176}$ R. Bucci, ${ }^{177}$ N. Dev, ${ }^{177}$ R. Goldouzian, ${ }^{177}$ M. Hildreth,${ }^{177}$ K. Hurtado Anampa, ${ }^{177}$ C. Jessop, ${ }^{177}$ D. J. Karmgard, ${ }^{177}$ K. Lannon, ${ }^{177}$ W. Li,${ }^{177}$ N. Loukas, ${ }^{177}$ N. Marinelli, ${ }^{177}$ I. Mcalister, ${ }^{177}$ F. Meng, ${ }^{177}$ C. Mueller, ${ }^{177}$ Y. Musienko, ${ }^{177,11}$ M. Planer, ${ }^{177}$ R. Ruchti,${ }^{177}$ P. Siddireddy, ${ }^{177}$ G. Smith, ${ }^{177}$ S. Taroni, ${ }^{177}$ M. Wayne, ${ }^{177}$ A. Wightman, ${ }^{177}$ M. Wolf, ${ }^{177}$ A. Woodard, ${ }^{177}$ J. Alimena, ${ }^{178}$ B. Bylsma, ${ }^{178}$ L. S. Durkin, ${ }^{178}$ B. Francis, ${ }^{178}$ C. Hill, ${ }^{178}$ W. Ji, ${ }^{178}$ A. Lefeld, ${ }^{178}$ T. Y. Ling, ${ }^{178}$ B. L. Winer, ${ }^{178}$ G. Dezoort, ${ }^{179}$ P. Elmer, ${ }^{179}$ J. Hardenbrook,${ }^{179}$ N. Haubrich,,${ }^{179}$ S. Higginbotham, ${ }^{179}$ A. Kalogeropoulos, ${ }^{179}$ S. Kwan, ${ }^{179}$ D. Lange, ${ }^{179}$ M. T. Lucchini, ${ }^{179}$ J. Luo, ${ }^{179}$ D. Marlow, ${ }^{179}$ K. Mei, ${ }^{179}$ I. Ojalvo, ${ }^{179}$ J. Olsen, ${ }^{179}$ C. Palmer, ${ }^{179}$ P. Piroué, ${ }^{179}$ D. Stickland, ${ }^{179}$ C. Tully, ${ }^{179}$ Z. Wang, ${ }^{179}$ S. Malik, ${ }^{180}$ S. Norberg, ${ }^{180}$ A. Barker, ${ }^{181}$ V. E. Barnes, ${ }^{181}$ S. Das, ${ }^{181}$ L. Gutay, ${ }^{181}$ M. Jones, ${ }^{181}$ A. W. Jung, ${ }^{181}$ A. Khatiwada, ${ }^{181}$ B. Mahakud, ${ }^{181}$ D. H. Miller, ${ }^{181}$ G. Negro, ${ }^{181}$ N. Neumeister, ${ }^{181}$ C. C. Peng, ${ }^{181}$ S. Piperov,${ }^{181}$ H. Qiu, ${ }^{181}$ J. F. Schulte, ${ }^{181}$ N. Trevisani, ${ }^{181}$ F. Wang, ${ }^{181}$ R. Xiao, ${ }^{181}$ W. Xie, ${ }^{181}$ T. Cheng, ${ }^{182}$ J. Dolen, ${ }^{182}$ N. Parashar, ${ }^{182}$ U. Behrens, ${ }^{183}$ K. M. Ecklund ${ }^{183}$ S. Freed, ${ }^{183}$ F. J. M. Geurts, ${ }^{183}$ M. Kilpatrick, ${ }^{183}$ Arun Kumar, ${ }^{183}$ W. Li, ${ }^{183}$ B. P. Padley, ${ }^{183}$ R. Redjimi, ${ }^{183}$ J. Roberts, ${ }^{183}$ J. Rorie, ${ }^{183}$ W. Shi, ${ }^{183}$ A. G. Stahl Leiton, ${ }^{183}$ Z. Tu, ${ }^{183}$ A. Zhang, ${ }^{183}$ A. Bodek, ${ }^{184}$ P. de Barbaro, ${ }^{184}$ R. Demina, ${ }^{184}$ J. L. Dulemba, ${ }^{184}$ C. Fallon, ${ }^{184}$ T. Ferbel, ${ }^{184}$ M. Galanti, ${ }^{184}$ A. Garcia-Bellido, ${ }^{184}$ O. Hindrichs, ${ }^{184}$ A. Khukhunaishvili, ${ }^{184}$ E. Ranken, ${ }^{184}$ R. Taus, ${ }^{184}$ B. Chiarito, ${ }^{185}$ J. P. Chou, ${ }^{185}$ A. Gandrakota, ${ }^{185}$ Y. Gershtein, ${ }^{185}$ E. Halkiadakis, ${ }^{185}$ A. Hart, ${ }^{185}$ M. Heindl, ${ }^{185}$ E. Hughes, ${ }^{185}$ S. Kaplan, ${ }^{185}$ I. Laflotte, ${ }^{185}$ A. Lath,${ }^{185}$ R. Montalvo, ${ }^{185}$ K. Nash, ${ }^{185}$ M. Osherson, ${ }^{185}$ H. Saka, ${ }^{185}$ S. Salur, ${ }^{185}$ S. Schnetzer, ${ }^{185}$ S. Somalwar, ${ }^{185}$ R. Stone, ${ }^{185}$ S. Thomas, ${ }^{185}$ H. Acharya,${ }^{186}$ A. G. Delannoy, ${ }^{186}$ S. Spanier,${ }^{186}$ O. Bouhali, ${ }^{187, y y y}$ M. Dalchenko, ${ }^{187}$ M. De Mattia, ${ }^{187}$ A. Delgado, ${ }^{187}$ S. Dildick, ${ }^{187}$ R. Eusebi, ${ }^{187}$ J. Gilmore,${ }^{187}$ T. Huang, ${ }^{187}$ T. Kamon, ${ }^{187, z z z}$ H. Kim, ${ }^{187}$ S. Luo, ${ }^{187}$ S. Malhotra, ${ }^{187}$ D. Marley, ${ }^{187}$ R. Mueller,${ }^{187}$ D. Overton, ${ }^{187}$ L. Perniè,${ }^{187}$ D. Rathjens, ${ }^{187}$ A. Safonov,${ }^{187}$ N. Akchurin, ${ }^{188}$ J. Damgov, ${ }^{188}$ F. De Guio, ${ }^{188}$ V. Hegde, ${ }^{188}$ S. Kunori, ${ }^{188}$ K. Lamichhane, ${ }^{188}$ S. W. Lee, ${ }^{188}$ T. Mengke, ${ }^{188}$ S. Muthumuni, ${ }^{188}$ T. Peltola, ${ }^{188}$ S. Undleeb,${ }^{188}$ I. Volobouev ${ }^{188}$ Z. Wang, ${ }^{188}$ A. Whitbeck, ${ }^{188}$ S. Greene, ${ }^{189}$ A. Gurrola, ${ }^{189}$ R. Janjam, ${ }^{189}$ W. Johns, ${ }^{189}$ C. Maguire, ${ }^{189}$ A. Melo, ${ }^{189}$ H. Ni, ${ }^{189}$ K. Padeken, ${ }^{189}$ F. Romeo, ${ }^{189}$ P. Sheldon, ${ }^{189}$ S. Starko, ${ }^{189}$ S. Tuo, ${ }^{189}$ J. Velkovska, ${ }^{189}$ M. Verweij, ${ }^{189}$ M. W. Arenton, ${ }^{190}$ P. Barria,${ }^{190}$ B. Cox, ${ }^{190}$ G. Cummings, ${ }^{190}$ J. Hakala, ${ }^{190}$ R. Hirosky, ${ }^{190}$ M. Joyce, ${ }^{190}$ A. Ledovskoy, ${ }^{190}$ C. Neu, ${ }^{190}$ B. Tannenwald,${ }^{190}$ Y. Wang, ${ }^{190}$ E. Wolfe, ${ }^{190}$ F. Xia, ${ }^{190}$ R. Harr, ${ }^{191}$ P. E. Karchin, ${ }^{191}$ N. Poudyal, ${ }^{191}$ J. Sturdy, ${ }^{191}$ P. Thapa, ${ }^{191}$ T. Bose, ${ }^{192}$ J. Buchanan, ${ }^{192}$ C. Caillol, ${ }^{192}$ D. Carlsmith, ${ }^{192}$ S. Dasu, ${ }^{192}$ I. De Bruyn, ${ }^{192}$ L. Dodd, ${ }^{192}$ F. Fiori, ${ }^{192}$ C. Galloni, ${ }^{192}$ H. He,${ }^{192}$ M. Herndon, ${ }^{192}$ A. Hervé, ${ }^{192}$ U. Hussain, ${ }^{192}$ P. Klabbers, ${ }^{192}$ A. Lanaro, ${ }^{192}$ A. Loeliger, ${ }^{192}$ K. Long, ${ }^{192}$ R. Loveless, ${ }^{192}$

J. Madhusudanan Sreekala, ${ }^{192}$ D. Pinna, ${ }^{192}$ T. Ruggles, ${ }^{192}$ A. Savin, ${ }^{192}$ V. Sharma, ${ }^{192}$ W. H. Smith, ${ }^{192}$ D. Teague, ${ }^{192}$ S. Trembath-reichert, ${ }^{192}$ and N. Woods ${ }^{192}$

(CMS Collaboration)

\author{
${ }^{1}$ Yerevan Physics Institute, Yerevan, Armenia \\ ${ }^{2}$ Institut für Hochenergiephysik, Wien, Austria \\ ${ }^{3}$ Institute for Nuclear Problems, Minsk, Belarus \\ ${ }^{4}$ Universiteit Antwerpen, Antwerpen, Belgium \\ ${ }^{5}$ Vrije Universiteit Brussel, Brussel, Belgium \\ ${ }^{6}$ Université Libre de Bruxelles, Bruxelles, Belgium \\ ${ }^{7}$ Ghent University, Ghent, Belgium \\ ${ }^{8}$ Université Catholique de Louvain, Louvain-la-Neuve, Belgium
}




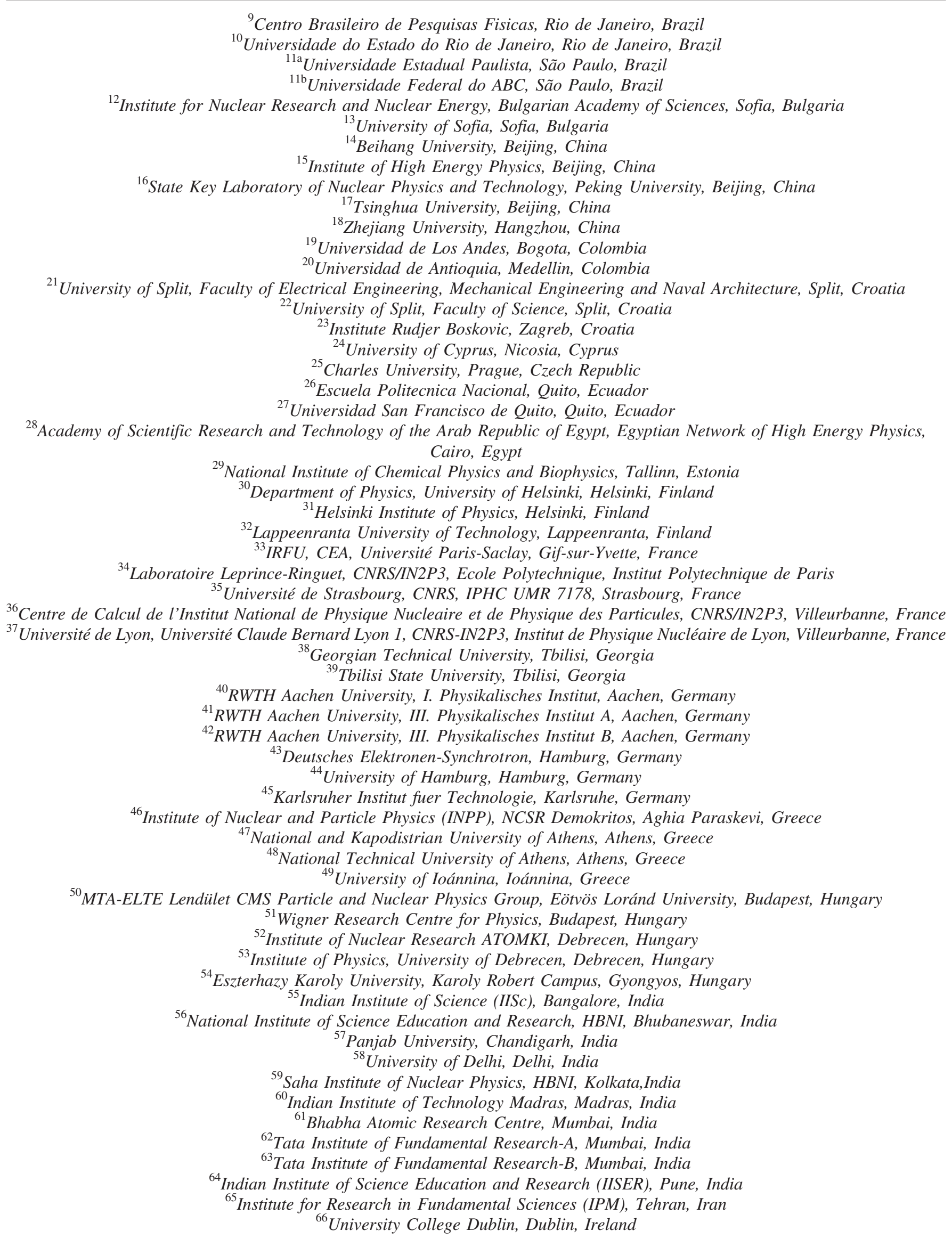




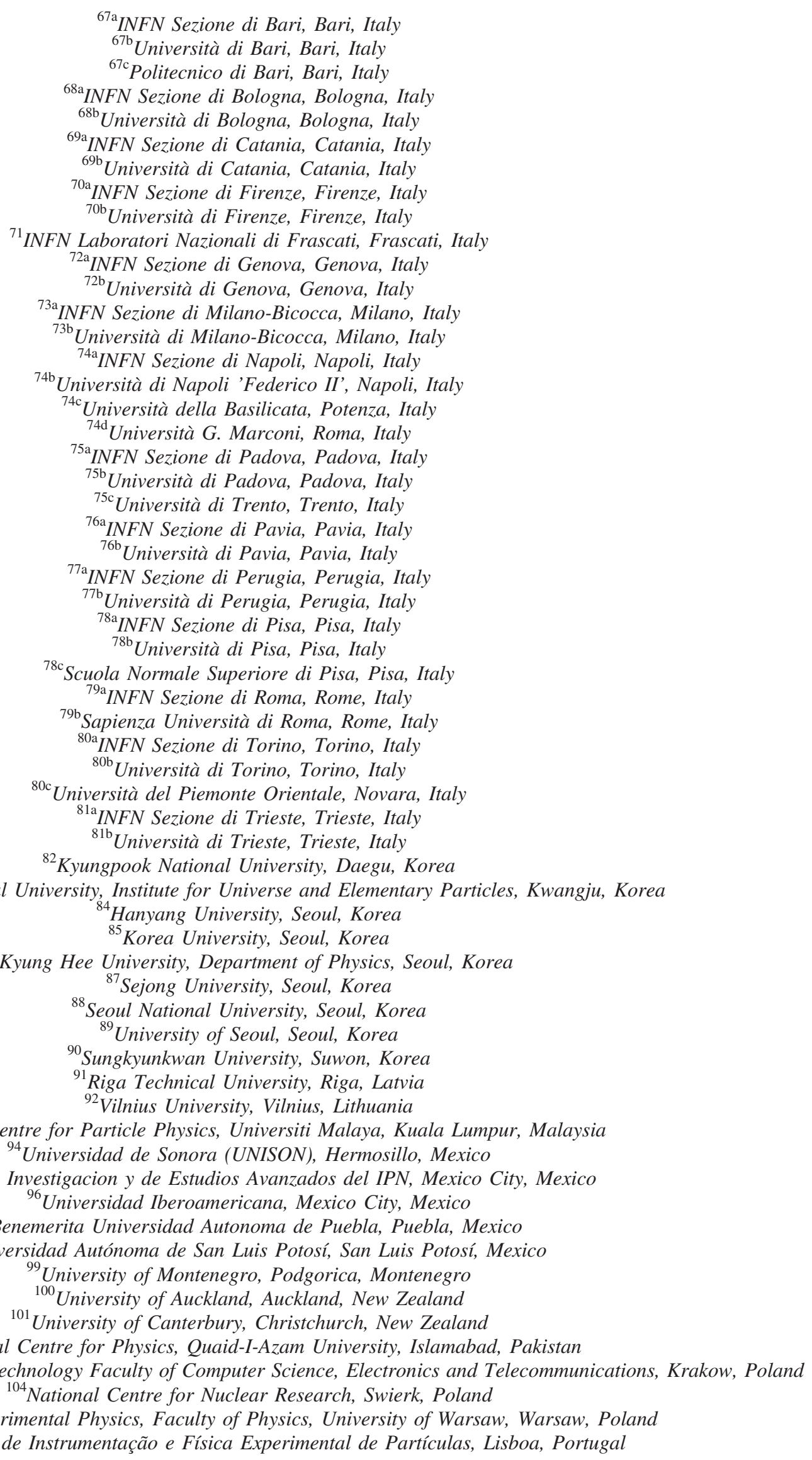


${ }^{107}$ Joint Institute for Nuclear Research, Dubna, Russia

${ }^{108}$ Petersburg Nuclear Physics Institute, Gatchina (St. Petersburg), Russia

${ }^{109}$ Institute for Nuclear Research, Moscow, Russia

${ }^{110}$ Institute for Theoretical and Experimental Physics named by A.I. Alikhanov of NRC 'Kurchatov Institute', Moscow, Russia

${ }^{111}$ Moscow Institute of Physics and Technology, Moscow, Russia

${ }^{112}$ National Research Nuclear University 'Moscow Engineering Physics Institute' (MEPhI), Moscow, Russia

${ }^{113}$ P.N. Lebedev Physical Institute, Moscow, Russia

${ }^{114}$ Skobeltsyn Institute of Nuclear Physics, Lomonosov Moscow State University, Moscow, Russia

${ }^{115}$ Novosibirsk State University (NSU), Novosibirsk, Russia

${ }^{116}$ Institute for High Energy Physics of National Research Centre 'Kurchatov Institute', Protvino, Russia

${ }^{117}$ National Research Tomsk Polytechnic University, Tomsk, Russia

${ }^{118}$ Tomsk State University, Tomsk, Russia

${ }^{119}$ University of Belgrade: Faculty of Physics and VINCA Institute of Nuclear Sciences, Belgrade, Serbia

${ }^{120}$ Centro de Investigaciones Energéticas Medioambientales y Tecnológicas (CIEMAT), Madrid, Spain

${ }^{121}$ Universidad Autónoma de Madrid, Madrid, Spain

${ }^{122}$ Universidad de Oviedo, Instituto Universitario de Ciencias y Tecnologías Espaciales de Asturias (ICTEA), Oviedo, Spain

${ }^{123}$ Instituto de Física de Cantabria (IFCA), CSIC-Universidad de Cantabria, Santander, Spain

${ }^{124}$ University of Colombo, Colombo, Sri Lanka

${ }^{125}$ University of Ruhuna, Department of Physics, Matara, Sri Lanka

${ }^{126}$ CERN, European Organization for Nuclear Research, Geneva, Switzerland

${ }^{127}$ Paul Scherrer Institut, Villigen, Switzerland

${ }^{128}$ ETH Zurich-Institute for Particle Physics and Astrophysics (IPA), Zurich, Switzerland

${ }^{129}$ Universität Zürich, Zurich, Switzerland

${ }^{130}$ National Central University, Chung-Li, Taiwan

${ }^{131}$ National Taiwan University (NTU), Taipei, Taiwan

${ }^{132}$ Chulalongkorn University, Faculty of Science, Department of Physics, Bangkok, Thailand

${ }^{133}$ Çukurova University, Physics Department, Science and Art Faculty, Adana, Turkey

${ }^{134}$ Middle East Technical University, Physics Department, Ankara, Turkey

${ }^{135}$ Bogazici University, Istanbul, Turkey

${ }^{136}$ Istanbul Technical University, Istanbul, Turkey

${ }^{137}$ Istanbul University, Istanbul, Turkey

${ }^{138}$ Institute for Scintillation Materials of National Academy of Science of Ukraine, Kharkov, Ukraine

${ }^{139}$ National Scientific Center, Kharkov Institute of Physics and Technology, Kharkov, Ukraine

${ }^{140}$ University of Bristol, Bristol, United Kingdom

${ }^{141}$ Rutherford Appleton Laboratory, Didcot, United Kingdom

${ }^{142}$ Imperial College, London, United Kingdom

${ }^{143}$ Brunel University, Uxbridge, United Kingdom

${ }^{144}$ Baylor University, Waco, Texas, USA

${ }^{145}$ Catholic University of America, Washington, DC, USA

${ }^{146}$ The University of Alabama, Tuscaloosa, Alabama, USA

${ }^{147}$ Boston University, Boston, Massachusetts, USA

${ }^{148}$ Brown University, Providence, Rhode Island, USA

${ }^{149}$ University of California at Davis, Davis, California, USA

${ }^{150}$ University of California at Los Angeles, Los Angeles, California, USA

${ }^{151}$ University of California at Riverside, Riverside, California, USA

${ }^{152}$ University of California at San Diego, La Jolla, California, USA

${ }^{153}$ University of California at Santa Barbara-Department of Physics, Santa Barbara, California, USA

${ }^{154}$ California Institute of Technology, Pasadena, California, USA

${ }^{155}$ Carnegie Mellon University, Pittsburgh, Pennsylvania, USA

${ }^{156}$ University of Colorado at Boulder, Boulder, Colorado, USA

${ }^{157}$ Cornell University, Ithaca, New York, USA

${ }^{158}$ Fermi National Accelerator Laboratory, Batavia, Illinois, USA

${ }^{159}$ University of Florida, Gainesville, Florida, USA

${ }^{160}$ Florida International University, Miami, Florida, USA

${ }^{161}$ Florida State University, Tallahassee, Florida, USA

${ }^{162}$ Florida Institute of Technology, Melbourne, Florida, USA

${ }^{163}$ University of Illinois at Chicago (UIC), Chicago, Illinois, USA

${ }^{164}$ The University of Iowa, Iowa City, Iowa, USA

${ }^{165}$ Johns Hopkins University, Baltimore, Maryland, USA

${ }^{166}$ The University of Kansas, Lawrence, Kansas, USA 
${ }^{167}$ Kansas State University, Manhattan, Kansas, USA

${ }^{168}$ Lawrence Livermore National Laboratory, Livermore, California, USA

${ }^{169}$ University of Maryland, College Park, Maryland, USA

${ }^{170}$ Massachusetts Institute of Technology, Cambridge, Massachusetts, USA

${ }^{171}$ University of Minnesota, Minneapolis, Minnesota, USA

${ }^{172}$ University of Mississippi, Oxford, Mississippi, USA

${ }^{173}$ University of Nebraska-Lincoln, Lincoln, Nebraska, USA

${ }^{174}$ State University of New York at Buffalo, Buffalo, New York, USA

${ }^{175}$ Northeastern University, Boston, Massachusetts, USA

${ }^{176}$ Northwestern University, Evanston, Illinois, USA

${ }^{177}$ University of Notre Dame, Notre Dame, Indiana, USA

${ }^{178}$ The Ohio State University, Columbus, Ohio, USA

${ }^{179}$ Princeton University, Princeton, New Jersey, USA

${ }^{180}$ University of Puerto Rico, Mayaguez, Puerto Rico, USA

${ }^{181}$ Purdue University, West Lafayette, Indiana, USA

${ }^{182}$ Purdue University Northwest, Hammond, Indiana, USA

${ }^{183}$ Rice University, Houston, Texas, USA

${ }^{184}$ University of Rochester, Rochester, New York, USA

${ }^{185}$ Rutgers, The State University of New Jersey, Piscataway, New Jersey, USA

${ }^{186}$ University of Tennessee, Knoxville, Tennessee, USA

${ }^{187}$ Texas A\&M University, College Station, Texas, USA

${ }^{188}$ Texas Tech University, Lubbock, Texas, USA

${ }^{189}$ Vanderbilt University, Nashville, Tennessee, USA

${ }^{190}$ University of Virginia, Charlottesville, Virginia, USA

${ }^{191}$ Wayne State University, Detroit, Michigan, USA

${ }^{192}$ University of Wisconsin, Madison, Wisconsin, USA

${ }^{\mathrm{a}}$ Deceased

${ }^{\mathrm{b}}$ Also at Vienna University of Technology, Vienna, Austria.

${ }^{\mathrm{c}}$ Also at IRFU, CEA, Université Paris-Saclay, Gif-sur-Yvette, France.

${ }^{\mathrm{d}}$ Also at Universidade Estadual de Campinas, Campinas, Brazil.

${ }^{\mathrm{e}}$ Also at Federal University of Rio Grande do Sul, Porto Alegre, Brazil.

${ }^{\mathrm{f}}$ Also at UFMS, Campo Grande, Brazil.

${ }^{\mathrm{g}}$ Also at Universidade Federal de Pelotas, Pelotas, Brazil.

${ }^{\mathrm{h}}$ Also at Université Libre de Bruxelles, Bruxelles, Belgium.

${ }^{\mathrm{i}}$ Also at University of Chinese Academy of Sciences, Bejing, China.

${ }^{j}$ Also at Institute for Theoretical and Experimental Physics named by A.I. Alikhanov of NRC 'Kurchatov Institute', Moscow, Russia.

${ }^{\mathrm{k}}$ Also at Joint Institute for Nuclear Research, Dubna, Russia.

${ }^{1}$ Also at British University in Egypt, Cairo, Egypt.

${ }^{\mathrm{m}}$ Also at Suez University, Suez, Egypt.

${ }^{\mathrm{n}}$ Also at Purdue University, West Lafayette, Indiana, USA.

${ }^{\circ}$ Also at Université de Haute Alsace, Mulhouse, France.

${ }^{\mathrm{p}}$ Also at Erzincan Binali Yildirim University, Erzincan, Turkey.

${ }^{\mathrm{q}}$ Also at CERN, European Organization for Nuclear Research, Geneva, Switzerland.

${ }^{\mathrm{r}}$ Also at RWTH Aachen University, III. Physikalisches Institut A, Aachen, Germany.

${ }^{\mathrm{s}}$ Also at University of Hamburg, Hamburg, Germany.

${ }^{\mathrm{t}}$ Also at Brandenburg University of Technology, Cottbus, Germany.

"Also at Institute of Physics, University of Debrecen, Debrecen, Hungary.

${ }^{v}$ Also at Institute of Nuclear Research ATOMKI, Debrecen, Hungary.

${ }^{w}$ Also at MTA-ELTE Lendület CMS Particle and Nuclear Physics Group, Eötvös Loránd University, Budapest, Hungary.

${ }^{\mathrm{x}}$ Also at IIT Bhubaneswar, Bhubaneswar, India.

${ }^{y}$ Also at Institute of Physics, Bhubaneswar, India.

${ }^{\mathrm{z}}$ Also at Shoolini University, Solan, India.

${ }^{\text {aa }}$ Also at University of Hyderabad, Hyderabad, India.

${ }^{\mathrm{bb}}$ Also at University of Visva-Bharati, Santiniketan, India.

${ }^{c c}$ Also at Isfahan University of Technology, Isfahan, Iran.

${ }^{\text {dd }}$ Also at INFN Sezione di Bari, Università di Bari, Politecnico di Bari, Bari, Italy.

${ }^{e e}$ Also at Italian National Agency for New Technologies, Energy and Sustainable Economic Development.

${ }^{\mathrm{ff}}$ Also at Centro Siciliano di Fisica Nucleare e di Struttura Della Materia, Catania, Italy.

${ }^{\text {gg }}$ Also at Scuola Normale e Sezione dell'INFN, Pisa, Italy. 
${ }^{\text {hh }}$ Also at Riga Technical University, Riga, Latvia.

ii Also at Malaysian Nuclear Agency, MOSTI, Kajang, Malaysia.

${ }^{j j}$ Also at Consejo Nacional de Ciencia y Tecnología, Mexico City, Mexico.

${ }^{\mathrm{kk}}$ Also at Warsaw University of Technology, Institute of Electronic Systems, Warsaw, Poland.

${ }^{11}$ Also at Institute for Nuclear Research, Moscow, Russia.

${ }^{\mathrm{mm}}$ Also at National Research Nuclear University 'Moscow Engineering Physics Institute' (MEPhI), Moscow, Russia.

${ }^{\mathrm{nn}}$ Also at St. Petersburg State Polytechnical University, St. Petersburg, Russia.

${ }^{\circ o}$ Also at University of Florida, Gainesville, Florida, USA.

${ }^{\mathrm{pp}}$ Also at Imperial College, London, United Kingdom.

${ }^{\mathrm{qq}}$ Also at P.N. Lebedev Physical Institute, Moscow, Russia.

${ }^{\text {rr }}$ Also at California Institute of Technology, Pasadena, California, USA.

${ }^{\text {ss }}$ Also at Budker Institute of Nuclear Physics, Novosibirsk, Russia.

"Also at Faculty of Physics, University of Belgrade, Belgrade, Serbia.

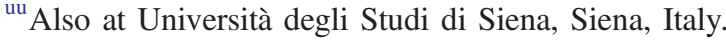

${ }^{v v}$ Also at INFN Sezione di Pavia, Università di Pavia, Pavia, Italy.

${ }^{\text {ww }}$ Also at National and Kapodistrian University of Athens, Athens, Greece.

${ }^{\mathrm{xx}}$ Also at Universität Zürich, Zurich, Switzerland.

${ }^{\text {yy }}$ Also at Stefan Meyer Institute for Subatomic Physics, Vienna, Austria.

${ }^{\mathrm{zz}}$ Also at Burdur Mehmet Akif Ersoy University, Burdur, Turkey.

${ }^{\text {aaa }}$ Also at Adiyaman University, Adiyaman, Turkey.

${ }^{b b b}$ Also at Şırnak University, Şırnak, Turkey.

${ }^{c c c}$ Also at Tsinghua University, Beijing, China.

ddd Also at Beykent University, Istanbul, Turkey.

${ }^{e e e}$ Also at Istanbul Aydin University, Istanbul, Turkey.

${ }^{\text {fff }}$ Also at Mersin University, Mersin, Turkey.

${ }^{\text {ggg }}$ Also at Piri Reis University, Istanbul, Turkey.

${ }^{\text {hhh }}$ Also at Gaziosmanpasa University, Tokat, Turkey.

iii Also at Ozyegin University, Istanbul, Turkey.

${ }^{\mathrm{ijj}}$ Also at Izmir Institute of Technology, Izmir, Turkey.

${ }^{k k k}$ Also at Marmara University, Istanbul, Turkey.

${ }^{111}$ Also at Kafkas University, Kars, Turkey.

${ }^{\mathrm{mmm}}$ Also at Istanbul Bilgi University, Istanbul, Turkey.

${ }^{\mathrm{nnn}}$ Also at Hacettepe University, Ankara, Turkey.

${ }^{\text {oоo }}$ Also at Vrije Universiteit Brussel, Brussel, Belgium.

${ }^{p p p}$ Also at School of Physics and Astronomy, University of Southampton, Southampton, United Kingdom.

${ }^{\mathrm{qqq}}$ Also at IPPP Durham University.

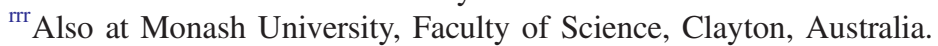

${ }^{\text {sss }}$ Also at Bethel University, St. Paul, Minneapolis, USA.

tttAlso at Karamanoğlu Mehmetbey University, Karaman, Turkey.

uшu Also at Bingol University, Bingol, Turkey.

${ }^{\mathrm{vvv}}$ Also at Georgian Technical University, Tbilisi, Georgia.

${ }^{w w w}$ Also at Sinop University, Sinop, Turkey.

${ }^{\mathrm{xxx}}$ Also at Mimar Sinan University, Istanbul, Istanbul, Turkey.

${ }^{\text {yyy }}$ Also at Texas A\&M University at Qatar, Doha, Qatar.

${ }^{\text {zzz }}$ Also at Kyungpook National University, Daegu, Korea. 